\title{
Analytical Multimodal Network Approach for 2-D Arrays of Planar Patches/Apertures Embedded in a Layered Medium
}

\author{
Raúl Rodríguez-Berral, Francisco Mesa, Fellow, IEEE, and Francisco Medina, Fellow, IEEE
}

\begin{abstract}
A fully analytical multimodal equivalent circuit is presented for the modeling of the scattering of an obliquely incident plane wave by a two-dimensional (2-D) periodic array of metallic patches (or apertures in a metallic screen) embedded in a layered medium. The topology of the equivalent network is rigorously derived in the analysis and all the network parameters are given in closed form. In contrast with the previously reported explicit circuit models, the proposed approach accounts for dynamical effects over a very wide frequency range, which enables the application of the model to a great variety of situations. The key advantages of the reported multimodal network representation are its analytical nature, its extremely low-computational cost and that the physical phenomena involved in the scattering can be easily understood in terms of transmission line and lumped circuital reasonings.
\end{abstract}

Index Terms-Electromagnetic scattering by periodic structures, equivalent circuits, frequency-selective surfaces (FSS).

\section{INTRODUCTION}

$\mathbf{T}$ HE STUDY of the electromagnetic properties of one dimensional (1-D) and two-dimensional (2-D) periodic distributions of planar metallic scatterers (or their complementary structures consisting of apertures in thin metal screens) embedded in layered dielectric media (see Fig. 1) is a classical topic in the microwave/antenna [1]-[5] and infrared [6]-[8] literature. In fact, this is the generic description of the widely used spatial filtering structures called frequency-selective surfaces (FSS) [9]-[13]. The study of other related devices such as reflectarrays [14], [15], artificial impedance surfaces [16], or resonant cavity antennas [17] would also benefit from the modeling of the class of structures considered in this paper. The study of this kind of problems also has a long tradition in the optics and physics communities. Indeed, in recent years, a lot of attention has been paid to 1-D and 2-D metallic

Manuscript received March 27, 2014; revised December 20, 2014; accepted February 09, 2015. Date of publication February 24, 2015; date of current version May 01, 2015. This work was supported in part by the Spanish Ministerio de Economía y Competitividad and European Union FEDER funds (Projects TEC2010-16948, TEC2013-41913-P, and Consolider CSD200800066) and in part by the Spanish Junta de Andalucía (Project P12-TIC-1435).

R. Rodríguez-Berral and F. Mesa are with the Microwaves Group, Department of Applied Physics 1, ETS de Ingeniería Informática, Universidad de Sevilla, 41012 Seville, Spain (e-mail: rrberral@us.es; mesa@us.es).

F. Medina is with the Department of Electronics and Electromagnetism, Faculty of Physics, Universidad de Sevilla, 41012 Seville, Spain (e-mail: medina@us.es).

Color versions of one or more of the figures in this paper are available online at http://ieeexplore.iee.org.

Digital Object Identifier 10.1109/TAP.2015.2406885 periodic structures in connection with the huge interest raised by extraordinary transmission, metasurfaces, and related problems [18]-[23]. A good review of the combined interest of physicists and engineers on this topic can be found in [24].

Although nowadays the analysis and design of 2-D periodic arrays are frequently carried out by means of general-purpose commercial electromagnetic solvers (or in-house specificpurpose numerical codes), the availability of analytical or quasi-analytical solutions adds a very convenient physical insight and provides apparent computational advantages for analysis/synthesis purposes. A good example of the usefulness of such analytical tools is found, for instance, in those optimization procedures that require the analysis of thousands of different structures in the corresponding parameters space. In this situation, the analytical expressions not only give numerical advantages but also provide a good insight in the searching of good initial guesses. Thus, a lot of effort has been devoted to the obtaining of approximate analytical solutions for a variety of geometries. Many of these solutions were determined in the frame of equivalent networks and other related approaches [1], [2], [4], [5], [25]-[37].

In this frame, and thanks to the periodicity of the structure and the excitation, the scattering problem under consideration can be posed in terms of a general waveguide discontinuity problem. An extensive literature has been developed to find the appropriate elements and topology of the equivalent network for a variety of such discontinuities [1], [38]-[42]. More recently, this standpoint has proved to be very useful for the treatment of extraordinary transmission in periodic structures [43], [44], planar metallic gratings [45], and FSS-like systems [46]-[50]. From early, finding closed-form expressions for the network elements as well as deriving an appropriate network topology was found to be an inherently complex task, especially for wide-band applications. Thus, most of the network topologies are based on heuristic reasonings that require either an a priori knowledge of the response of the structure in a wide frequency range (namely, a complete full-wave problem has to be solved in advance) or they work properly only under certain limits of validity (in the long wavelength regime, for instance). If the networks are employed beyond these limits, it is usually assumed that the same topology still works but then the circuit parameters have to be obtained via a fitting procedure that requires the aid of full-wave commercial electromagnetic simulators or specific in-house software. This fitting procedure certainly extends the applicability of the equivalent 


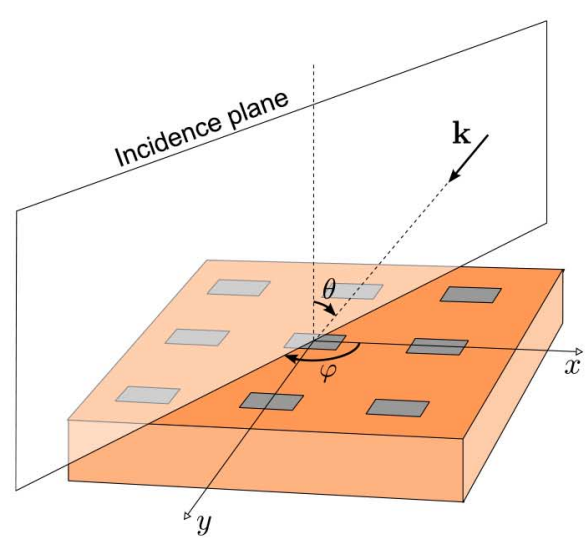

(a)

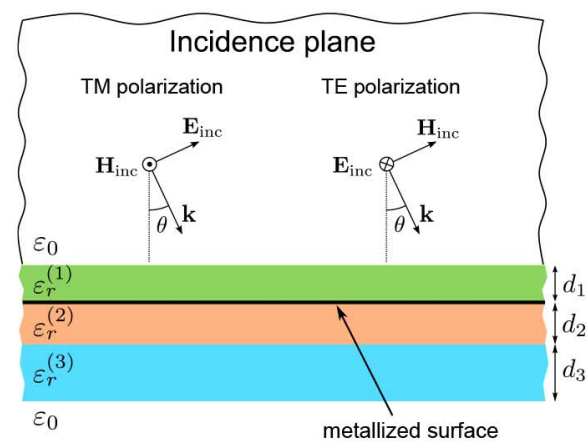

(b)
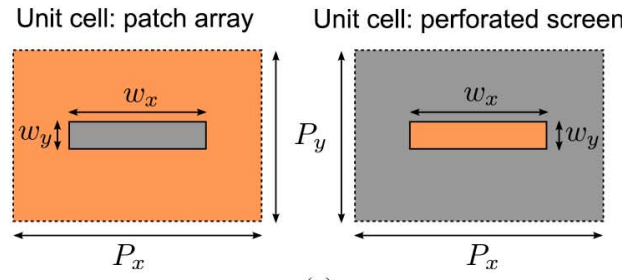

(c)

Fig. 1. Structure under study: 2-D periodic distribution of rectangular scatterers (metallic patches or apertures in the metallic screen) embedded in a layered dielectric medium. (a) Plane wave impinges obliquely on the structure with its wavevector characterized by the angles $\theta$ and $\varphi$. (b) Incidence-plane cut showing the possible TE and TM polarizations of the incident wave. Each dielectric layer is characterized by its permittivity $\varepsilon_{i}=\varepsilon_{0} \varepsilon_{r}^{(i)}$ and thickness $d_{i}$. (c) Cross views of the unit cells for the two cases (patches/apertures) considered in this work. The boundaries of the unit cells are, in general, periodic boundary conditions accounting for field polarization and the phase shift imposed by the wave vector orientation.

network approach to many situations involving scatterers of complex shape and/or multiresonant nature as well as to multilayered environment [48]. Nevertheless, the circuit models thus obtained are usually limited to relatively low frequencies or are of narrow-band nature. Those models need to be "redesigned" if the geometry of the scatterers and/or the layered environment change, in such a way that some kind of "relay-race" procedure has to be used to cover a wide frequency band. Another typical drawback of the conventional circuit models available in the literature comes from the inclusion of dielectric slabs. The effect of the dielectric layers surrounding the metalized interface is often partially incorporated in the equivalent network by means of an effective dielectric medium approach [15], [46].
This procedure cannot take into account complex and exotic effects such as those described, for instance, in [51] and [52], where the role of the surface waves guided by the dielectric slabs is essential. A very useful alternative to the use of complex equivalent networks, still providing accurate enough analytical expressions for the transmission/reflection coefficients, is based on the use of homogenization procedures [35], [53], [54]; however, it is well known that this approach should be limited to the long wavelength regime.

In [43], [45], and [47], very accurate and wideband circuit models were reported to account for dynamic/distributed effects that had not been incorporated in previous equivalent network representations. Thus, very complex spectra, RayleighWood anomalies, and extraordinary transmission/reflection peaks were accurately reproduced by those models. However, in these papers, as well as in many others on this topic, some of the essential parameters of the circuit models had to be retrieved from full-wave numerical simulations at a few arbitrarily chosen frequency points. Moreover, to a certain extent, the topology of the circuits was obtained using heuristic reasonings. In search of a systematic procedure to obtain the topology of the equivalent network, the authors of this paper first studied the simpler case of 1-D grating problems. The analysis of the TE/TM oblique incidence in the principal planes of the structure for a 1-D grating embedded in a layered medium was reported in [55]. In that work, the topology of the equivalent network that actually accounts for the electromagnetic behavior of the structures was rigorously derived along with closed-form expressions for the involved circuit parameters. [Two rigorous method of moments (MoM) analysis of this 1-D periodic structure for conical incidence, which is a central topic to this paper, can be found in [56] and in a recent contribution [57]]. In a preliminary step, the study in [55] was applied to the 2-D case in [58]. In this work, this last study is considerably extended in order to find a systematic procedure to obtain the appropriate and explicit topology of the multimodal equivalent network as well as closed-form expressions for all the elements of this network. Arbitrary angles of incidence (conical incidence) are also considered in this theoretical frame. It will be shown that our analytical solution, apart from preventing the need for numerical full-wave simulations, completely captures the physics of the problem over a very wide frequency region that extends even within the grating lobe regime (free-space wavelength shorter than the period of the structure).

The structure under analysis in this work is a 2-D periodic distribution of rectangular scatterers (metallic patches) or apertures in a metallic screen (rectangular slots) that is embedded in a layered dielectric medium (see Fig. 1). The incident field is assumed to be an obliquely impinging time-harmonic plane wave (either TE or TM polarized in the incidence plane) characterized by its angular frequency $\omega$, its wavenumber in free space $k_{0}=\omega / c$, and arbitrary incidence angles $\theta$ and $\varphi$. The presence of an eventual ground plane in the structure can also be incorporated in the analysis in a straightforward way. The case of a slot-based FSS surrounded by semi-infinite homogeneous dielectrics is first considered in Section II-A. After this study, it is discussed how to incorporate the existence of a layered environment (and an eventual ground plane). The analysis 
of patch-based FSS is briefly considered in Section II-B following a similar rationale as that of slot-based FSS. Some considerations on the practical and efficient computation of the equivalent-network elements are discussed in Section III. Next, Section IV shows the strategy to deal with the conical incidence in terms of auxiliary problems, in which the electric field of the incident wave is parallel to the principal planes of the structure. Some numerical validations and examples are given in Section V, and finally, the conclusion of this work is briefly exposed in Section VI.

\section{Formal DERIVATION OF THE EQUiVAlENT NETWORKS}

The periodic nature of the problem under study allows for a Floquet analysis of the structure in Fig. 1(a). The scattering analysis can then be restricted to the unit cell shown in Fig. 1(c). This unit-cell problem can alternatively be seen as a discontinuity problem in a rectangular waveguide with periodic boundary conditions. The harmonics of the Floquet analysis can thus be regarded as the modes of the generalized waveguide [59], so that a microwave network approach [39] can be applied. Next, the derivation of the circuit models is carried out separately for slot- and patch-based FSS.

\section{A. Slot-Based FSS}

The oblique incidence of a plane wave that impinges on a slot-based FSS is studied in this section (see Fig. 1). In most practical cases, the impinging wave will be either the $\mathrm{TM}_{00}$ or the $\mathrm{TE}_{00}$ harmonic but, in principle, it can be any of the harmonics of the periodic structure, i.e., any of the modes of the generalized waveguide (unit cell) shown in Fig. 2(a). From this waveguide standpoint, our task is to find the equivalent network that accounts for the scattering of the incident mode, as sketched in Fig. 2(b). Although the incident harmonic is scattered at both the dielectric interfaces and the perforated metallic screen, only the latter involves coupling with different harmonics. For this reason, the problem of a perforated-screen discontinuity between two surrounding semi-infinite homogeneous dielectrics shown in Fig. 3 is first considered (this is our fundamental discontinuity problem). The presence of dielectric slabs is left to a further step of the analysis.

The Floquet expansion of the transverse ( $x, y$ components) electric and magnetic field at the discontinuity $(z=0)$ can be written as

$$
\begin{aligned}
\mathbf{E}(x, y)= & (1+R) \mathbf{e}_{0}(x, y)+\sum_{h}^{\prime} V_{h} \mathbf{e}_{h}(x, y) \\
\mathbf{H}^{(1)}(x, y)= & Y_{0}^{(1)}(1-R)\left[\hat{\mathbf{z}} \times \mathbf{e}_{0}(x, y)\right] \\
& -\sum_{h}^{\prime} Y_{h}^{(1)} V_{h}\left[\hat{\mathbf{z}} \times \mathbf{e}_{h}(x, y)\right] \\
\mathbf{H}^{(2)}(x, y)= & Y_{0}^{(2)}(1+R)\left[\hat{\mathbf{z}} \times \mathbf{e}_{0}(x, y)\right] \\
& +\sum_{h}^{\prime} Y_{h}^{(2)} V_{h}\left[\hat{\mathbf{z}} \times \mathbf{e}_{h}(x, y)\right]
\end{aligned}
$$

where subindex $h=0$ stands here for the incident harmonic, $R$ is the reflection coefficient of the incident wave (this incident

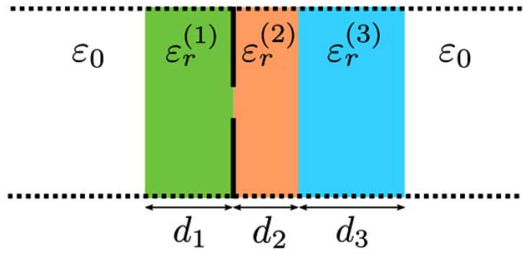

(a)

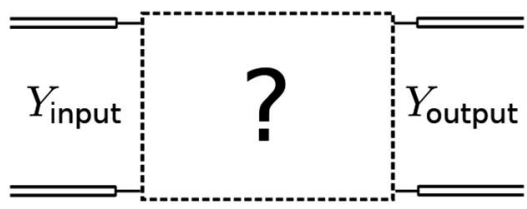

(b)

Fig. 2. (a) Waveguide problem to model the scattering of a plane wave incident on a periodically perforated screen embedded in a three-layer medium (a generalized layered medium is assumed in the analysis). (b) Our problem is to find the topology and values of the equivalent network that accounts for the scattering of the incident plane wave.

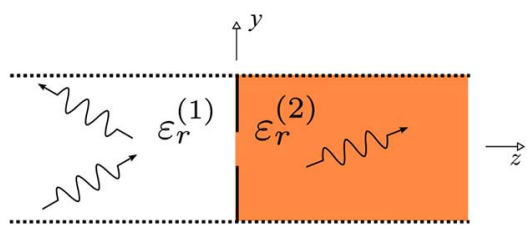

Fig. 3. Fundamental waveguide discontinuity problem (diaphragm) associated with a perforated-screen located between two homogeneous semi-infinite media under plane-wave oblique incidence. For simplicity in the figure, the incidence plane is taken as the principal $y z$ plane.

wave has been conveniently normalized), $V_{h}$ are the unknown coefficients of the electric field expansion, the $(i)$ super-indexes refer to the medium and the prime in the series indicates that the incident harmonic is excluded from the summation, which comprises the remaining infinite set of harmonics. Each harmonic $h$ is associated with a pair of integer numbers $n \mathrm{~m}$ and its normalized transverse field profile, $\mathbf{e}_{h}(x, y)$, and other associated magnitudes are

$$
\begin{aligned}
\mathbf{e}_{h}(x, y) & =\frac{\mathrm{e}^{-\mathrm{j} \mathbf{k}_{\mathrm{t}, h} \cdot \boldsymbol{\rho}}}{\sqrt{P_{x} P_{y}}} \hat{\mathbf{e}}_{h} \quad[\boldsymbol{\rho}=x \hat{\mathbf{x}}+y \hat{\mathbf{y}}] \\
\mathbf{k}_{\mathrm{t}, h} & =k_{x n} \hat{\mathbf{x}}+k_{y m} \hat{\mathbf{y}} \\
& =\left(k_{x 0}+k_{n}\right) \hat{\mathbf{x}}+\left(k_{y 0}+k_{m}\right) \hat{\mathbf{y}}
\end{aligned}
$$

with

$$
\begin{gathered}
k_{x 0}=k_{0} \sin \theta \cos \varphi \\
k_{y 0}=k_{0} \sin \theta \sin \varphi \\
k_{n}=\frac{2 \pi n}{P_{x}} \\
k_{m}=\frac{2 \pi m}{P_{y}}
\end{gathered}
$$


( $k_{0}$ is the vacuum wavenumber). The modal admittances $Y_{h}^{(i)}$ are given by

$$
Y_{h}^{(i)}=\frac{H_{\mathrm{t}, h}}{E_{\mathrm{t}, h}}=\frac{1}{\eta^{(i)}} \begin{cases}k^{(i)} / \beta_{n m}^{(i)}, & \text { TM harmonics } \\ \beta_{n m}^{(i)} / k^{(i)}, & \text { TE harmonics }\end{cases}
$$

where

$$
\begin{aligned}
& k^{(i)}=\sqrt{\varepsilon_{r}^{(i)}} k_{0} \\
& \beta_{h}^{(i)}=\sqrt{\varepsilon_{r}^{(i)} k_{0}^{2}-\left|\mathbf{k}_{\mathrm{t}, h}\right|^{2}} \\
& \eta^{(i)}=\frac{\eta_{0}}{\sqrt{\varepsilon_{r}^{(i)}}}
\end{aligned}
$$

with $\eta_{0}$ being the impedance of free space.

If $\mathbf{E}_{a}(x, y)$ is the actual electric field in the aperture, (1) must then represent the Fourier expansion of this field, whose expansion coefficients are given by

$$
\begin{aligned}
1+R & =\frac{1}{\sqrt{P_{x} P_{y}}} \widetilde{\mathbf{E}}_{\mathrm{a}}\left(\mathbf{k}_{\mathrm{t}, 0}\right) \cdot \hat{\mathbf{e}}_{0} \\
V_{h} & =\frac{1}{\sqrt{P_{x} P_{y}}} \widetilde{\mathbf{E}}_{\mathrm{a}}\left(\mathbf{k}_{\mathrm{t}, h}\right) \cdot \hat{\mathbf{e}}_{h}
\end{aligned}
$$

where the symbol $\sim$ represents Fourier transform with respect to $x, y$. The coefficients of the electric field expansion are then related by $\left(1+R \equiv V_{0}\right)$

$$
\frac{V_{h}}{N_{h}}=\frac{1+R}{N_{0}}
$$

where

$$
N_{h}=\widetilde{\mathbf{E}}_{\mathrm{a}}\left(\mathbf{k}_{\mathrm{t}, h}\right) \cdot \hat{\mathbf{e}}_{h} .
$$

Equation (19) already suggests a topology of the equivalent network. If we associate a transmission line voltage signal $V_{h}$ to each harmonic, then (19) would be consistent with an equivalent network, in which the transmission line representing each harmonic is connected in parallel with all the others through a transformer with turn ratio $N_{h}$. The continuity of the Poynting vector through the aperture (ap.)

$$
\iint_{\text {ap. }}\left[\mathbf{E}_{\mathrm{a}}^{*} \times\left(\mathbf{H}_{2}-\mathbf{H}_{1}\right)\right] \cdot \hat{\mathbf{z}} \mathrm{d} x \mathrm{~d} y=0
$$

will give us the equation for the equivalent-network currents that is consistent with this interpretation. Thus, introducing (2) and (3) into (21) it is obtained that

$$
\begin{aligned}
& N_{0}^{*} Y_{0}^{(1)}(1-R)-\sum_{h}^{\prime} N_{h}^{*} Y_{h}^{(1)} V_{h} \\
& \quad=N_{0}^{*} Y_{0}^{(2)}(1+R)+\sum_{h}^{\prime} N_{h}^{*} Y_{h}^{(2)} V_{h} .
\end{aligned}
$$

If the characteristic admittance of each transmission line is taken as the corresponding modal admittance in (13), $\pm Y_{h}^{(i)} V_{h}$ can be identified with the transmission line currents (the \pm sign

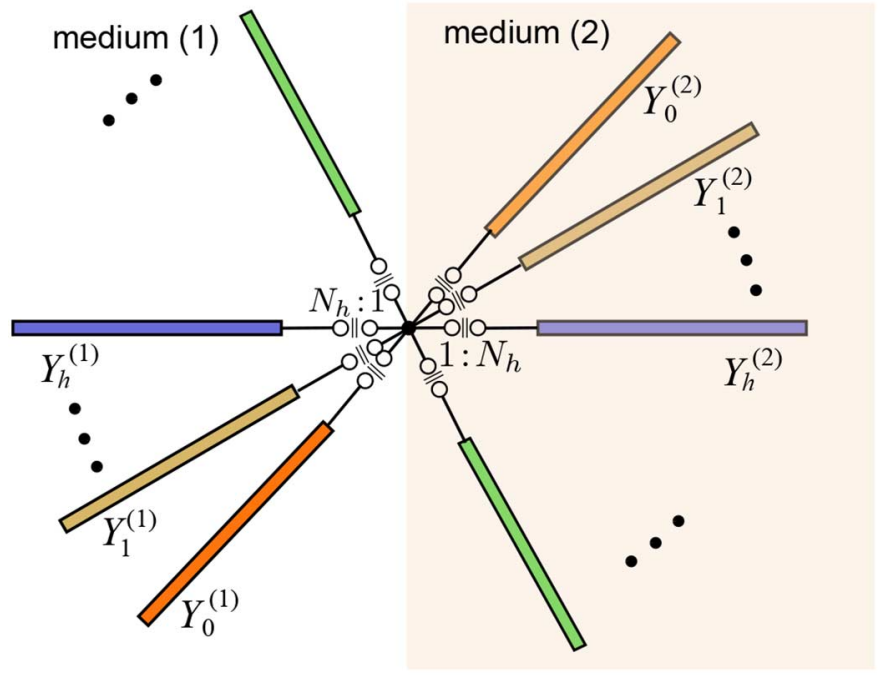

(a)

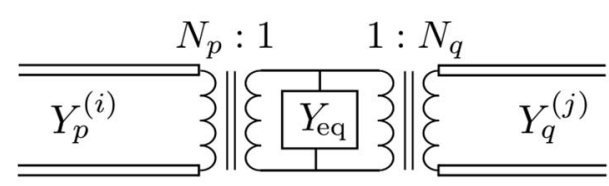

(b)

Fig. 4. (a) "Top" view of the multimodal network representation of the scattering problem associated with a slot-based FSS surrounded by two homogeneous media. The symbol - $॥$ - - stands here for a transformer. (b) "Standard" view of the transmission line problem representing the scattering from the $p$-harmonic in medium $(i)$ to the $q$-harmonic in medium $(j)$. The equivalent admittance $Y_{\text {eq }}$ accounts for the global effect of the parallel-connected transmission lines corresponding to all the other harmonics.

corresponds to signals propagating along the positive/negative $z$ direction) and (22) becomes

$$
\sum_{h} N_{h}^{*} I_{h}^{(1)}=\sum_{h} N_{h}^{*} I_{h}^{(2)} .
$$

The above derivation thus leads unambiguously to a general multimode equivalent transmission-line network where every harmonic $h$ in each medium $(i)$ is represented by a transmission line with characteristic admittance equal to its transverse wave admittance, $Y_{h}^{(i)}$, which is connected in parallel through its corresponding transformer $N_{h}$ to all the other lines associated with the remaining harmonics. This network is schematically depicted in Fig. 4(a). Thus, for an input $p$ harmonic from medium $(i)$ and an output $q$ harmonic in medium $(j)$, the following scattering parameters can be obtained from the equivalent network represented in Fig. 4(b)

$$
\begin{aligned}
S_{i(p), i(p)} & =\frac{\left|N_{p}\right|^{2} Y_{p}^{(i)}-\left|N_{q}\right|^{2} Y_{q}^{(j)}-Y_{\mathrm{eq}}}{\left|N_{p}\right|^{2} Y_{p}^{(i)}+\left|N_{q}\right|^{2} Y_{q}^{(j)}+Y_{\mathrm{eq}}} \\
S_{j(q), i(p)} & =\frac{2 N_{q} N_{p}^{*} Y_{p}^{(i)}}{\left|N_{p}\right|^{2} Y_{p}^{(i)}+\left|N_{q}\right|^{2} Y_{q}^{(j)}+Y_{\mathrm{eq}}}
\end{aligned}
$$

where the equivalent admittance $Y_{\text {eq }}$ is given by

$$
Y_{\text {eq }}=\sum_{h}\left|N_{h}\right|^{2}\left[Y_{h}^{(1)}+Y_{h}^{(2)}\right]-\left|N_{p}\right|^{2} Y_{p}^{(i)}-\left|N_{q}\right|^{2} Y_{q}^{(j)}
$$




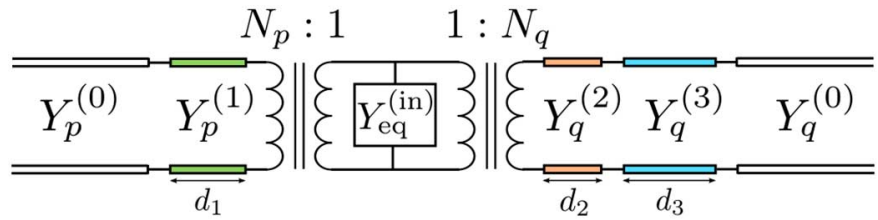

(a)

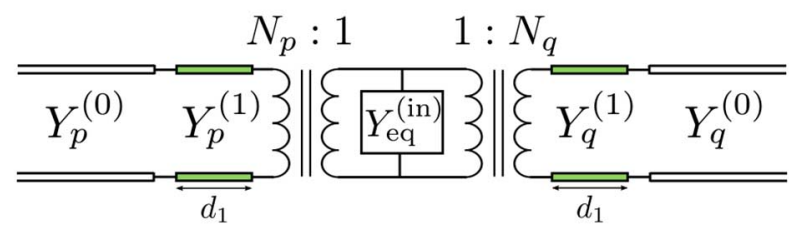

(b)
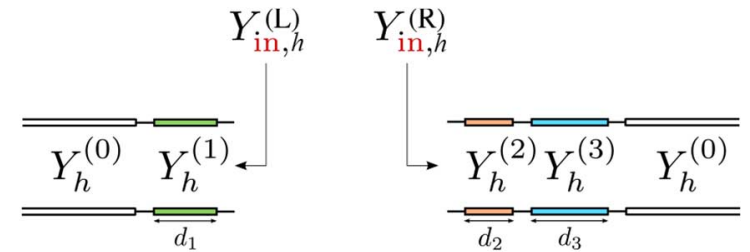

(c)

Fig. 5. Considering the multilayered dielectric environment in Figs. 1(b) and 2(a), this figure shows the equivalent network for the computation of the scattering parameters corresponding to: (a) harmonic $p$ and $q$ to the left- and right-hand side of the screen, respectively. (b) Harmonics $p$ and $q$, both at the left-hand side of the screen. (c) Definition of the input admittances associated with the $h$-harmonic.

and accounts for the contribution of all the other harmonics. In particular, the reflection coefficient for the $h=0$ harmonic can directly be obtained by introducing (2), (3), and (19) into (22) and solving for $R$, which yields

$$
R=\frac{\left|N_{0}\right|^{2} Y_{0}^{(1)}-\left|N_{0}\right|^{2} Y_{0}^{(2)}-Y_{\mathrm{eq}}}{\left|N_{0}\right|^{2} Y_{0}^{(1)}+\left|N_{0}\right|^{2} Y_{0}^{(2)}+Y_{\mathrm{eq}}}=S_{1(0), 1(0)} .
$$

Once the general network has been derived for an FSS placed between two homogeneous media, the generalization to a multilayered dielectric environment is relatively straightforward, since it just implies to replace the transmission lines in the model with a cascade of transmission line lengths, each one corresponding to a dielectric layer. This concept is represented in Fig. 5(a) and (b). The equivalent admittance appearing in these networks must be generalized from its original form in (26) by replacing the $Y_{h}^{(1)}$ and $Y_{h}^{(2)}$ admittances with the corresponding input admittances $Y_{\mathrm{in}, h}^{(\mathrm{L})}$ and $Y_{\mathrm{in}, h}^{(\mathrm{R})}$, which represent the input admittances to the left/right (L/R) cascade of dielectrics [see Fig. 5(c)]. For instance, for the case of Fig. 5(a), we have

$Y_{\mathrm{eq}}^{(\mathrm{in})}=\sum_{h}\left|N_{h}\right|^{2}\left[Y_{\mathrm{in}, h}^{(\mathrm{L})}+Y_{\mathrm{in}, h}^{(\mathrm{R})}\right]-\left|N_{p}\right|^{2} Y_{\mathrm{in}, p}^{(\mathrm{L})}-\left|N_{q}\right|^{2} Y_{\mathrm{in}, q}^{(\mathrm{R})}$.

For any other configuration of layers, the changes should be evident at the light of the above figure. The relative simplicity of this procedure is due to the fact that the different harmonics do not couple at the interfaces between dielectric layers. At this point, it is worth mentioning that the presence of an electric/magnetic plane can be readily introduced

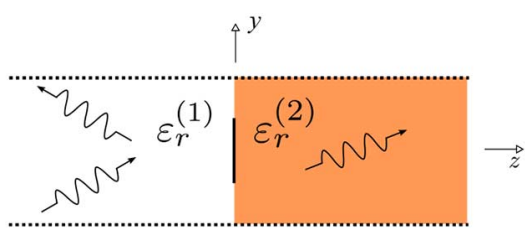

Fig. 6. Fundamental waveguide discontinuity problem (planar obstacle) associated with the patch-based FSS surrounded by two semi-infinite homogeneous media.

in the definition of the input admittances by transforming conveniently this short/open-circuit load along the corresponding cascade of transmission lines. Thus, the presented formalism can rigorously take into account the presence of real or virtual electric/magnetic walls, even if they are in close proximity to the perforated conducting surface.

\section{B. Patch-Based FSS}

The scattering of a plane wave impinging on a periodic array of metallic scatterers embedded in a layered environment (i.e., the structure consisting on replacing the previous perforated conducting screen by its complementary version) can be treated in a similar way as in the previous section. The case of homogeneous dielectrics at both sides of the periodic screen is considered first (see Fig. 6). It should be noted that due to the presence of dielectrics, the present problem involving an array of patches is not dual (in the Babinet sense) to the perforated screen case.

The starting point for this derivation is again the transverse fields expansions in (1)-(3), but now the surface current density on the patches $\mathbf{J}_{\mathrm{p}}(x, y)$ is employed instead of the aperture field. The coefficients of the field expansion are related to the surface current through the magnetic field jump condition at the discontinuity plane $(z=0)$

$$
\mathbf{J}_{\mathrm{p}}(x, y)=\hat{\mathbf{z}} \times\left[\mathbf{H}^{(2)}(x, y)-\mathbf{H}^{(1)}(x, y)\right] .
$$

Introducing the magnetic field expansions (2) and (3) into (29), the right-hand side of this last equation represents the Fourier expansion of the surface current density. Identifying the coefficients of the Fourier expansion and after some manipulations, it is found that

$$
\begin{aligned}
& N_{h}^{*}\left[Y_{h}^{(1)}+Y_{h}^{(2)}\right] V_{h}=N_{0}^{*}\left[-(1-R) Y_{0}^{(1)}+T Y_{0}^{(2)}\right] \\
& N_{p}^{*}\left[Y_{p}^{(1)}+Y_{p}^{(2)}\right] V_{p}=N_{q}^{*}\left[Y_{q}^{(1)}+Y_{q}^{(2)}\right] V_{q}
\end{aligned}
$$

with $p, q \neq 0, T=1+R$, and

$$
N_{h}=\left[\widetilde{\mathbf{J}}_{\mathrm{p}}^{*}\left(\mathbf{k}_{\mathrm{t}, h}\right) \cdot \hat{\mathbf{e}}_{h}\right]^{-1} \text {. }
$$

Although not as straightforward as (19), expressions (30) and (31) can also be interpreted in terms of transmission-line currents. As sketched in Fig. 7(a), these equations suggest a parallel connection between the transmission lines representing a given harmonic with order $h$ at both sides of the screen, and also that each $h$-order subnetwork is connected in series with all the rest through its corresponding transformer-since, 


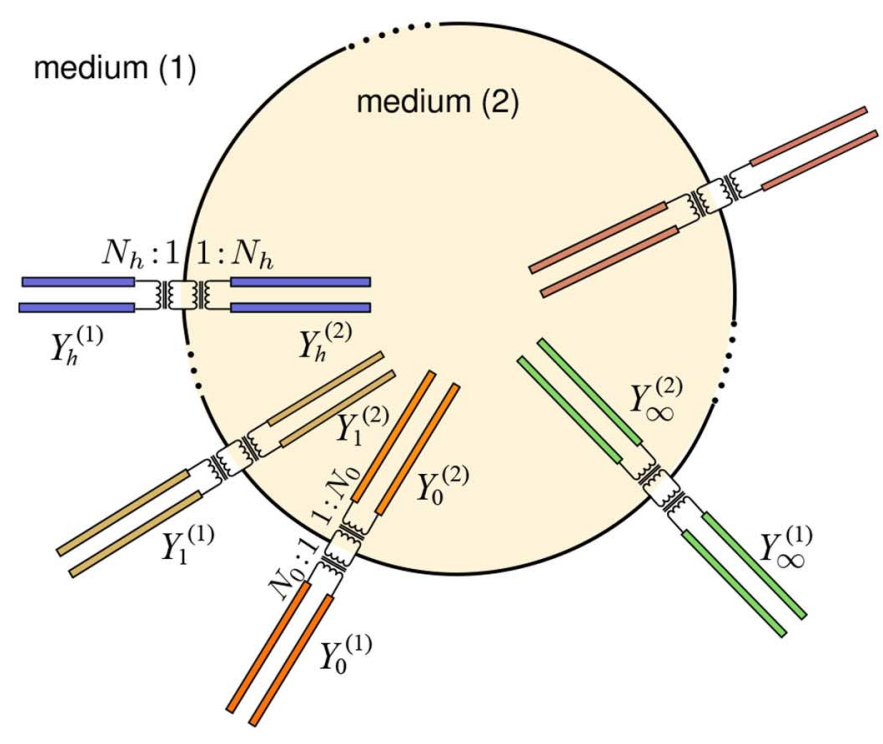

(a)

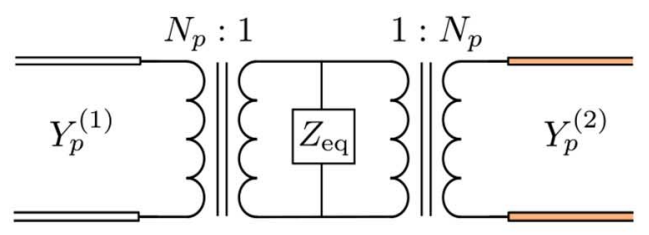

(b)

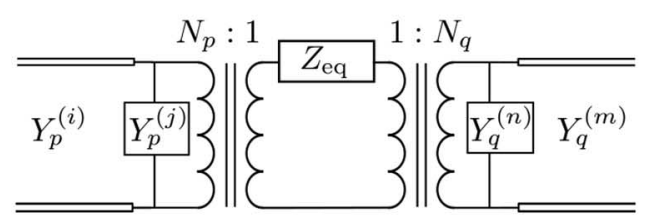

(c)

Fig. 7. (a) Multimodal equivalent network associated with a periodic array of metallic scatterers surrounded by two homogeneous media. (b) Equivalent circuit for the scattering of the $p$ th order harmonic. (c) Equivalent circuit for the transmission/reflection from harmonic $p$ in medium $(i)$ to harmonic $q$ in medium $(m)$, with $p \neq q, j \neq i$ and $n \neq m$ (for instance, if $i=1$ and $m=2$, then $j=2$ and $n=1$ ).

according to (30) and (31), the total current entering the $h$ th subnetwork through its associated transformer is the same for all the harmonics. The corresponding equation for the voltages can be obtained from the following weak form of the boundary condition for the electric field on the metallic surface

$$
\iint_{\text {patch }} \mathbf{J}_{\mathrm{p}}^{*} \cdot \mathbf{E} \mathrm{d} x \mathrm{~d} y=0 .
$$

Introducing (1) into this equation, it is found that

$$
\frac{1+R}{N_{0}}+\sum_{h}^{\prime} \frac{V_{h}}{N_{h}}=0
$$

which is fully consistent with the interpretation shown in Fig. 7(a). The particular forms of this general equivalent network for the scattering of a given $p$-order harmonic at both sides of the discontinuity and for harmonic $p$ in medium $(i)$ to harmonic $q$ in medium $(m)$ are represented in Fig. 7(b) and (c), respectively. In these figures, the equivalent impedance $Z_{\text {eq }}$ is given by the following sum:

$$
Z_{\text {eq }}=\sum_{h}^{\prime} \frac{1}{\left|N_{h}\right|^{2}\left(Y_{h}^{(1)}+Y_{h}^{(2)}\right)}
$$

where the prime indicates that the sum extends to all the other harmonics [i.e., harmonic $p$ is excluded in the case of Fig. 7(b), and both the $p$ and $q$ harmonics are excluded for Fig. 7(c)]. The application of the standard procedures of the transmissionline theory [60] to this multimodal equivalent network would provide the expressions for the generalized scattering parameters. Thus, the same formal expression as in (24) is again obtained for the reflection coefficient $S_{i(p), i(p)}$. The expression for $S_{j(q), i(p)}$ can be obtained, for instance, by cascading the $\mathrm{ABCD}$ matrices [60] corresponding to the equivalent-circuit elements in Fig. 7(c). Finally, the presence of a layered environment can again be introduced via transmission-line lengths into each transmission line in the equivalent network.

\section{Computation of Network Elements}

The topologies of the transmission-line networks described in previous sections have been rigorously deduced and, hence, their corresponding scattering parameters are formally equivalent to those of the original FSS problems. Although these multimodal equivalent networks might be considered by themselves a relevant theoretical contribution with interesting potential applications, their practical implementation requires some important considerations which are addressed next.

First, the transformers turns ratios $N_{h}$ in (20) and (32) have been expressed in terms of the electric field at the apertures or the surface current density on the patches but, certainly, these quantities are not known a priori (the knowledge of these quantities implies to have already solved the scattering problem). Nevertheless, under certain circumstances, the spatial profile of the aperture field or patch current is not expected to change significantly with frequency, although the complex amplitude of such quantities does obviously change. In other words, in such circumstances, it is appropriate to assume the following factorization:

$$
\begin{aligned}
\mathbf{E}_{\mathrm{a}}(x, y ; \omega) & =F(\omega) \mathbf{E}_{\mathrm{a}}(x, y) \\
\mathbf{J}_{\mathrm{p}}(x, y ; \omega) & =G(\omega) \mathbf{J}_{\mathrm{p}}(x, y) .
\end{aligned}
$$

The assumption of a frequency-independent spatial profile in the above quantities (equivalent to using just one basis function in the standard application of the MoM) makes that the same factorization translates to all the transformer turns ratios $N_{h}$, and thus, their frequency dependence cancels out in the final expressions of the scattering parameters. Therefore, the $N_{h}$ parameters can be computed using the assumed spatial profile of the aperture field or patch current and ignoring their frequency-dependent amplitudes $F(\omega)$ and $G(\omega)$. The frequency dependence of the scattering parameters would then come from the input TE/TM admittances, which are explicitly known. For rectangular apertures/patches under oblique incidence in the principal planes of the structure $(\varphi=0$ or $\varphi=90^{\circ}$ ), approximate closed-form expressions for the aperture field and patch surface current profiles are available [61], 
which results in a fully analytical circuit model that provides good numerical results as shown in Section V. Unfortunately, the factorization in (36) and (37) cannot be considered a good approximation in the general and important case of conical incidence $\left(\varphi \neq 0, \varphi \neq 90^{\circ}\right)$, with independence of the simplicity of the patch or aperture geometry. A superposition strategy is proposed in Section IV to deal with such general situation.

A second important consideration is that the proposed equivalent networks involve an infinite number of transmission lines, corresponding to the infinite number of harmonics excited at the periodic screen discontinuity. In the computation of the scattering parameters, it means that the equivalent admittance/impedance is given as an infinite double series. In order to overcome this drawback, the same strategy previously used by the authors in [45], [47], and [55] can be employed here. The basic idea is to consider separately the contribution of low- and high-order harmonics, following the same rationale used in [34] concerning the distinction of "accessible" and "localized" modes (the "localized" modes in [34] can here be identified with the high-order harmonics). For a given $n m$ harmonic operating well below its cutoff frequency (high-order harmonic)

$$
\begin{aligned}
k_{x n} & =k_{0}+k_{n} \approx k_{n} \\
k_{y m} & =k_{0}+k_{m} \approx k_{m}
\end{aligned}
$$

it is possible to make the following approximations:

$$
\begin{aligned}
\mathbf{k}_{t, h} & \approx k_{n} \hat{\mathbf{x}}+k_{m} \hat{\mathbf{y}}=\mathbf{k}_{h} \\
\left|\mathbf{k}_{t, h}\right| & \approx \sqrt{k_{n}^{2}+k_{m}^{2}}=k_{h} \\
\hat{\mathbf{k}}_{h} & =\frac{\mathbf{k}_{h}}{k_{h}} \\
\hat{\mathbf{e}}_{h}^{\text {ho }} & = \begin{cases}\hat{\mathbf{k}}_{h}, & \text { TM harmonics } \\
\hat{\mathbf{k}}_{h} \times \hat{\mathbf{z}}, & \text { TE harmonics }\end{cases}
\end{aligned}
$$

where the label "ho" (high order) indicates in the following that $k_{x n}, k_{y m}$ are substituted by $k_{n}, k_{m}$, respectively. The above approximation also allows us to write

$$
\beta_{h}^{(i)} \approx-\mathrm{j} k_{h}=-\mathrm{j} \sqrt{k_{n}^{2}+k_{m}^{2}}
$$

and therefore, the wave admittances can be approximated as

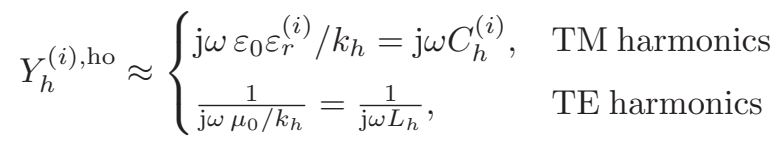

where the coefficients $C_{h}^{(i)}$ and $L_{h}$ can be interpreted, respectively, as frequency-independent capacitances and inductances associated with sufficiently high-order TM and TE harmonics in medium $(i)$. When a layered environment is present, each TM high-order cascaded transmission line appears in the model as a given frequency-independent "input capacitance," $C_{\mathrm{in}, h}^{(\mathrm{L} / \mathrm{R})}$. For high-order TE harmonics, note that the inductive admittance in (45) does not depend on the medium, but only on the order $h$. Therefore, the corresponding "input inductance" is not affected by the presence of the dielectric layers and is given directly by $L_{h}$. According to the above discussion, the global contribution of all the high-order harmonics consists of a parallel $L C$ tank for a slot-based FSS and as a series $L C$ tank for patch-based FSS. More specifically, given certain $M$ such that $\forall|n|,|m|>M$ the conditions (38) and (39) are satisfied, it is possible to write the equivalent admittance (28) for the slot-based problem as

$$
\begin{aligned}
Y_{\mathrm{eq}}^{(\mathrm{in})} \approx & \mathrm{j} \omega C_{\mathrm{ho}}+\frac{1}{\mathrm{j} \omega L_{\mathrm{ho}}} \\
& +\sum_{|n|,|m| \leq M}^{\prime}\left|N_{n m}\right|^{2}\left[Y_{\mathrm{in}, h}^{(\mathrm{L})}+Y_{\mathrm{in}, h}^{(\mathrm{R})}\right]
\end{aligned}
$$

where

$$
\begin{aligned}
C_{\mathrm{ho}} & =\sum_{|n, m|=M+1}^{\infty}\left|N_{n m, \mathrm{TM}}^{\mathrm{ho}}\right|^{2}\left[C_{\mathrm{in}, n m}^{(\mathrm{L})}+C_{\mathrm{in}, n m}^{(\mathrm{R})}\right] \\
\frac{1}{L_{\mathrm{ho}}} & =\sum_{|n, m|=M+1}^{\infty} \frac{2\left|N_{n m, \mathrm{TE}}^{\mathrm{ho}}\right|^{2}}{L_{n m}} .
\end{aligned}
$$

For the periodic array of patches, it is equivalently found that

$$
\begin{aligned}
Z_{\mathrm{eq}}^{(\mathrm{in})} \approx & \mathrm{j} \omega L_{\mathrm{ho}}+\frac{1}{\mathrm{j} \omega C_{\mathrm{ho}}} \\
& +\sum_{|n|,|m| \leq M}^{\prime}\left\{\left|N_{n m}\right|^{2}\left[Y_{\mathrm{in}, h}^{(\mathrm{L})}+Y_{\mathrm{in}, h}^{(\mathrm{R})}\right]\right\}^{-1}
\end{aligned}
$$

with

$$
\begin{aligned}
\frac{1}{C_{\mathrm{ho}}} & =\sum_{|n, m|=M+1}^{\infty} \frac{1}{\left|N_{n m, \mathrm{TM}}^{\mathrm{ho}}\right|^{2}\left[C_{\mathrm{in}, n m}^{(\mathrm{L})}+C_{\mathrm{in}, n m}^{(\mathrm{R})}\right]} \\
L_{\mathrm{ho}} & =\sum_{|n, m|=M+1}^{\infty} \frac{L_{n m}}{2\left|N_{n m, \mathrm{TE}}^{\mathrm{ho}}\right|^{2}} .
\end{aligned}
$$

As an example, for the layered medium at the left-hand side of Fig. 2(a), the "input capacitance" corresponding to the $\mathrm{nm}$ harmonic is

$$
C_{\mathrm{in}, n m}^{(\mathrm{L})}=\frac{\varepsilon_{0} \varepsilon_{r}^{(1)}}{k_{n m}} \frac{\varepsilon_{r}^{(0)}+\varepsilon_{r}^{(1)} \tanh \left(k_{n m} d_{1}\right)}{\varepsilon_{r}^{(1)}+\varepsilon_{r}^{(0)} \tanh \left(k_{n m} d_{1}\right)}
$$

with $k_{n m}=\sqrt{k_{n}^{2}+k_{m}^{2}}$.

The resulting topology of the final circuit models is illustrated in Fig. 8. It should be noted that although the obtaining of the $C_{\mathrm{ho}}$ and $L_{\mathrm{ho}}$ parameters involves the computation of infinite double series, these series are independent of both frequency and angle of incidence. This feature is computationally very advantageous for the usual case of frequency and/or angle of incidence sweeps, since the series only need to be computed once. It is interesting to consider that, provided the highest frequency of interest is still low enough so that the case $M=$ 0 yields sufficiently accurately results, the proposed equivalent network would reduce to just two lumped elements: $C_{\mathrm{ho}}$ and $L_{\mathrm{ho}}$. This situation resembles the so-called homogenization procedure reported, for instance, in [35] and [54]. However, in 

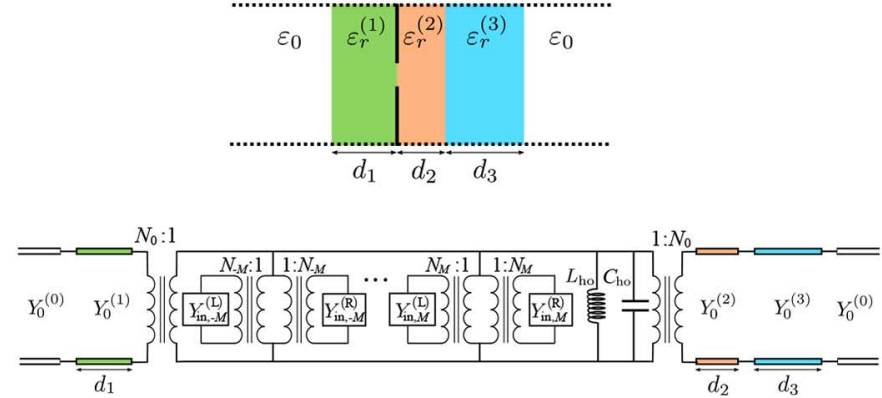

(a)

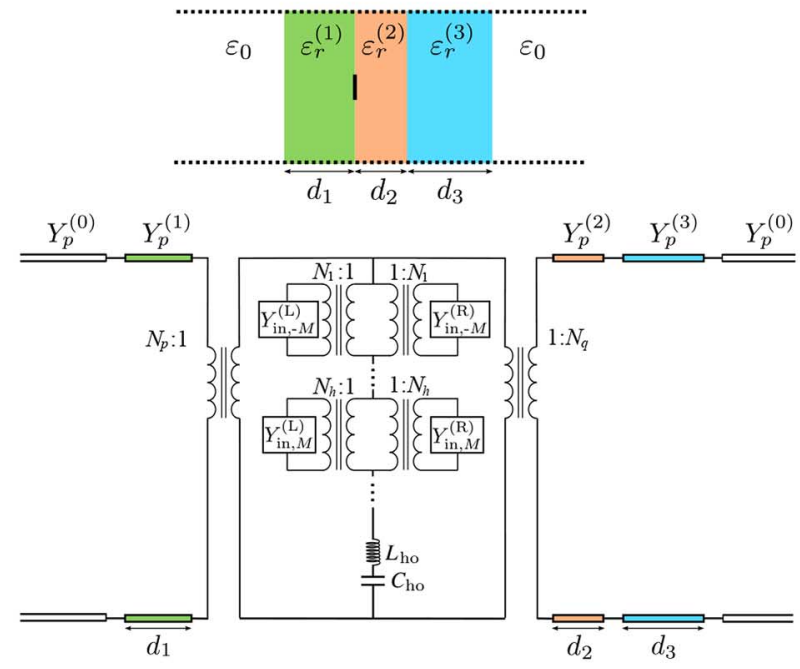

(b)

Fig. 8. Two examples of the final topology of the circuit models corresponding to the computation of the scattering parameters of the kind of structures studied in this paper. (a) Equivalent network to compute the zeroth-order harmonics scattering parameters of a perforated metallic screen. (b) Equivalent network to compute $S_{i(p), j(p)}$ for a patch-based FSS embedded in a layered medium.

the present $M=0$ case, our inductance and capacitance elements do take into account the presence of the layered dielectric environment, and also the eventual existence of ground planes. Taking values of $M \geq 1$ improves accuracy and extends the validity of the model to higher frequencies, including the diffraction regime ( $\lambda$ is smaller than $P_{x}$ and/or $P_{y}$ ), which is not considered by conventional circuit-like models.

\section{Superposition Strategy For CONICAL InCIDENCE}

It was already mentioned that for the general case of conical incidence (i.e., the incidence plane does not coincide with any of the principal planes of the structure-in our case, the $x=0$ and $y=0$ planes), the factorization in (36) and (37) is not a good assumption. Even if it is assumed that, approximately, the spatial/frequency dependence of each Cartesian component of the field/current spatial profile can be factorized as it was done in (36) and (37), namely

$$
\begin{aligned}
\mathbf{E}_{\mathrm{a}}(x, y ; \omega) & =F_{x}(\omega) E_{a, x}(x, y) \hat{\mathbf{x}}+F_{y}(\omega) E_{a, y}(x, y) \hat{\mathbf{y}} \\
\mathbf{J}_{\mathrm{p}}(x, y ; \omega) & =G_{x}(\omega) J_{p, x}(x, y) \hat{\mathbf{x}}+G_{y}(\omega) J_{p, y}(x, y) \hat{\mathbf{y}}
\end{aligned}
$$

the frequency factors affecting each of the Cartesian components are different and there is no a priori knowledge about the relationship between them. Hence, the very opportune frequency-dependent cancellation in the definition of the $N_{h}$ parameters derived from (36) and (37) would not be achieved in this case. Certainly, for incidence in the principal planes of the structures (as those treated in our previous works [55], [58]), the factorization shown in (36) and (37) is expected to work properly, since it is possible to write now the field/current profile as

$$
\begin{aligned}
\mathbf{E}_{\mathrm{a}}(x, y ; \omega) & =F(\omega) E_{a}(x, y) \hat{\mathbf{s}} \\
\mathbf{J}_{\mathrm{p}}(x, y ; \omega) & =G(\omega) J_{p}(x, y) \hat{\mathbf{s}}
\end{aligned}
$$

where $s$ stands for $x$ or $y$ indistinctly.

The extension of this procedure to plane-wave conical incidence would be very useful in order to cover such situations found in practical applications (for instance, a generic element of a reflectarray illuminated by a feeding horn). Our purpose now is then to pose the original conical-incidence problem into a form amenable to be solved by the method in this paper. The chosen solution is to define superpositions of incident plane waves (auxiliary problems) leading to patch/slot excitations for which the factorization given in (55) or (56) can be applied. This is expected to be approximately possible for specific combinations of TE and TM excitations yielding strictly $\hat{x}$-directed or $\hat{\mathbf{y}}$-directed tangential electric field over the slot or patch. From the complex amplitudes of the TE and TM transmitted and reflected waves in such auxiliary cases, the desired scattering parameters can be obtained by applying the superposition principle. The algebraic details are explained in what follows.

In general, any incident plane wave at angles $(\theta, \varphi)$ can be decomposed as a sum of incident TM and TE waves with those same incidence angles

$$
\mathbf{E}_{\mathrm{inc}}=\mathbf{E}_{\mathrm{inc}}^{\mathrm{TM}}+\mathbf{E}_{\mathrm{inc}}^{\mathrm{TE}}
$$

where the transverse (to the plane of incidence) fields in the above decomposition are given by

$$
\begin{aligned}
\mathbf{E}_{\text {inc }}^{\mathrm{TM}} & =E_{\text {inc }}^{\mathrm{TM}} \cos \varphi \hat{\mathbf{x}}+E_{\text {inc }}^{\mathrm{TM}} \sin \varphi \hat{\mathbf{y}} \\
\mathbf{E}_{\text {inc }}^{\mathrm{TE}} & =E_{\text {inc }}^{\mathrm{TE}} \sin \varphi \hat{\mathbf{x}}-E_{\text {inc }}^{\mathrm{TE}} \cos \varphi \hat{\mathbf{y}}
\end{aligned}
$$

and therefore

$$
\begin{aligned}
\mathbf{E}_{\mathrm{inc}}= & \left(E_{\mathrm{inc}}^{\mathrm{TM}} \cos \varphi+E_{\mathrm{inc}}^{\mathrm{TE}} \sin \varphi\right) \hat{\mathbf{x}} \\
& +\left(E_{\mathrm{inc}}^{\mathrm{TM}} \sin \varphi-E_{\mathrm{inc}}^{\mathrm{TE}} \cos \varphi\right) \hat{\mathbf{y}} .
\end{aligned}
$$

Thus, the case

$$
\begin{aligned}
E_{\text {inc }}^{\mathrm{TM}} & =E_{0}^{(x)} \cos \varphi \\
E_{\text {inc }}^{\mathrm{TE}} & =E_{0}^{(x)} \sin \varphi
\end{aligned}
$$

corresponds to an incident wave whose electric field is directed along $x$

$$
\mathbf{E}_{\text {inc }}=E_{0}^{(x)} \hat{\mathbf{x}}
$$

Similarly, the case

$$
\begin{aligned}
E_{\mathrm{inc}}^{\mathrm{TM}} & =E_{0}^{(y)} \sin \varphi \\
E_{\mathrm{inc}}^{\mathrm{TE}} & =-E_{0}^{(y)} \cos \varphi
\end{aligned}
$$


corresponds to an incident wave whose electric field is directed along $y$

$$
\mathbf{E}_{\text {inc }}=E_{0}^{(y)} \hat{\mathbf{y}} .
$$

The above two cases will be denoted as the $x$-problem and the $y$-problem, respectively. Now it is assumed that the spatial profiles of the aperture field (or patch current) excited at the screen by such incident fields are similar to the ones expected for incidence in the principal planes. For the $x$-problem, it means that the profiles are assumed to be similar to those for $\varphi=90^{\circ}$ and TE incidence or $\varphi=0$ and TM incidence. For the $y$-problem, the profiles are assumed to be similar to those for $\varphi=90^{\circ}$ and TM incidence or $\varphi=0$ and TE incidence. Since these problems satisfy the conditions that made it possible the factorizations (36) and (37), the procedures of the previous sections will be employed to deal with these auxiliary scattering problems. It leads to an equivalent network for the $x$-problem and a different one for the $y$-problem (the topologies are the same, but the value of the elements in the network are different as a consequence of the different spatial profiles). From these networks, it is then possible to compute the following auxiliary scattering parameters:

$$
\begin{array}{ll}
x \text {-problem }: & R_{x}^{\mathrm{TM}, \mathrm{TM}}, R_{x}^{\mathrm{TM}, \mathrm{TE}}, R_{x}^{\mathrm{TE}, \mathrm{TE}}, R_{x}^{\mathrm{TE}, \mathrm{TM}} \\
y \text {-problem }: & R_{y}^{\mathrm{TM}, \mathrm{TM}}, R_{y}^{\mathrm{TM}, \mathrm{TE}}, R_{y}^{\mathrm{TE}, \mathrm{TE}}, R_{y}^{\mathrm{TE}, \mathrm{TM}}
\end{array}
$$

where, for instance, $R_{x}^{\mathrm{TM}, \mathrm{TE}}$ stands for the reflection coefficient that relates the TM component of the output wave to the TE component of the input wave for the $x$ problem (incident electric field parallel to the $x$-axis). These auxiliary coefficients can now be combined to obtain the actual co-pol and cross-pol reflection coefficients for a pure TM or TE incident wave, as shown next.

\section{A. TM Incidence}

The incident electric field can be written in this case as

$$
\mathbf{E}^{\mathrm{TM}}=E_{0}^{\mathrm{TM}}(\cos \varphi \hat{\mathbf{x}}+\sin \varphi \hat{\mathbf{y}})=E_{0}^{(x)} \hat{\mathbf{x}}+E_{0}^{(y)} \hat{\mathbf{y}}
$$

with

$$
\begin{aligned}
& E_{0}^{(x)}=E_{0}^{\mathrm{TM}} \cos \varphi \\
& E_{0}^{(y)}=E_{0}^{\mathrm{TM}} \sin \varphi .
\end{aligned}
$$

Using now (61), (62), (64), and (65), the actual co-pol $\left(R_{\mathrm{TM}, \mathrm{TM}}\right)$ and cross-pol $\left(R_{\mathrm{TE}, \mathrm{TM}}\right)$ reflection coefficients are then given by

$$
\begin{aligned}
R_{\mathrm{TM}, \mathrm{TM}} E_{0}^{\mathrm{TM}}= & R_{x}^{\mathrm{TM}, \mathrm{TM}} E_{0}^{(x)} \cos \varphi+R_{x}^{\mathrm{TM}, \mathrm{TE}} E_{0}^{(x)} \sin \varphi \\
& +R_{y}^{\mathrm{TM}, \mathrm{TM}} E_{0}^{(y)} \sin \varphi-R_{y}^{\mathrm{TM}, \mathrm{TE}} E_{0}^{(y)} \cos \varphi \\
R_{\mathrm{TE}, \mathrm{TM}} E_{0}^{\mathrm{TM}}= & R_{x}^{\mathrm{TE}, \mathrm{TE}} E_{0}^{(x)} \sin \varphi+R_{x}^{\mathrm{TE}, \mathrm{TM}} E_{0}^{(x)} \cos \varphi \\
& -R_{y}^{\mathrm{TE}, \mathrm{TE}} E_{0}^{(y)} \cos \varphi+R_{y}^{\mathrm{TE}, \mathrm{TM}} E_{0}^{(y)} \sin \varphi .
\end{aligned}
$$

Finally, the substitution of (70) and (71) into the two last expressions gives the following expressions:

$$
\begin{aligned}
R_{\mathrm{TM}, \mathrm{TM}}= & R_{x}^{\mathrm{TM}, \mathrm{TM}} \cos ^{2} \varphi+R_{y}^{\mathrm{TM}, \mathrm{TM}} \sin ^{2} \varphi \\
& +\left[R_{x}^{\mathrm{TM}, \mathrm{TE}}-R_{y}^{\mathrm{TM}, \mathrm{TE}}\right] \sin \varphi \cos \varphi \\
R_{\mathrm{TE}, \mathrm{TM}}= & R_{x}^{\mathrm{TE}, \mathrm{TM}} \cos ^{2} \varphi+R_{y}^{\mathrm{TE}, \mathrm{TM}} \sin ^{2} \varphi \\
& +\left[R_{x}^{\mathrm{TE}, \mathrm{TE}}-R_{y}^{\mathrm{TE}, \mathrm{TE}}\right] \sin \varphi \cos \varphi .
\end{aligned}
$$

\section{B. TE Incidence}

In this case, the incident electric field can be written as

$$
\mathbf{E}^{\mathrm{TE}}=E_{0}^{\mathrm{TM}}(\sin \varphi \hat{\mathbf{x}}-\cos \varphi \hat{\mathbf{y}})=E_{0}^{(x)} \hat{\mathbf{x}}+E_{0}^{(y)} \hat{\mathbf{y}}
$$

with

$$
\begin{aligned}
& E_{0}^{(x)}=E_{0}^{\mathrm{TE}} \sin \varphi \\
& E_{0}^{(y)}=-E_{0}^{\mathrm{TE}} \cos \varphi .
\end{aligned}
$$

Using (61), (62), (64), and (65), the actual co-pol and cross-pol reflection coefficients are then given by

$$
\begin{aligned}
R_{\mathrm{TE}, \mathrm{TE}} E_{0}^{\mathrm{TE}}= & R_{x}^{\mathrm{TE}, \mathrm{TE}} E_{0}^{(x)} \sin \varphi+R_{x}^{\mathrm{TE}, \mathrm{TM}} E_{0}^{(x)} \cos \varphi \\
& -R_{y}^{\mathrm{TE}, \mathrm{TE}} E_{0}^{(y)} \cos \varphi+R_{y}^{\mathrm{TE}, \mathrm{TM}} E_{0}^{(y)} \sin \varphi
\end{aligned}
$$

$$
\begin{aligned}
R_{\mathrm{TM}, \mathrm{TE}} E_{0}^{\mathrm{TE}}= & R_{x}^{\mathrm{TM}, \mathrm{TM}} E_{0}^{(x)} \cos \varphi+R_{x}^{\mathrm{TM}, \mathrm{TE}} E_{0}^{(x)} \sin \varphi \\
& +R_{y}^{\mathrm{TM}, \mathrm{TM}} E_{0}^{(y)} \sin \varphi-R_{y}^{\mathrm{TM}, \mathrm{TE}} E_{0}^{(y)} \cos \varphi
\end{aligned}
$$

Finally, substituting (77) and (78) into the two last expressions leads to

$$
\begin{aligned}
R_{\mathrm{TE}, \mathrm{TE}}= & R_{x}^{\mathrm{TE}, \mathrm{TE}} \sin ^{2} \varphi+R_{y}^{\mathrm{TE}, \mathrm{TE}} \cos ^{2} \varphi \\
& +\left[R_{x}^{\mathrm{TE}, \mathrm{TM}}-R_{y}^{\mathrm{TE}, \mathrm{TM}}\right] \sin \varphi \cos \varphi \\
R_{\mathrm{TM}, \mathrm{TE}}= & R_{x}^{\mathrm{TM}, \mathrm{TE}} \sin ^{2} \varphi+R_{y}^{\mathrm{TM}, \mathrm{TE}} \cos ^{2} \varphi \\
& +\left[R_{x}^{\mathrm{TM}, \mathrm{TM}}-R_{y}^{\mathrm{TM}, \mathrm{TM}}\right] \sin \varphi \cos \varphi .
\end{aligned}
$$

\section{NUMERICAL RESULTS}

Different results are presented in this section to validate the suitability and degree of accuracy of the proposed circuit-like model. Our closed-form results are first compared with some examples reported in the literature that include highly accurate data computed by means of an ad hoc MoM or MoM/BI-RME (boundary integral-resonant mode expansion). Note that for this class of planar structures, the results calculated with a method of this type can be considered virtually "exact." Comparison examples with results obtained using a general-purpose commercial electromagnetic simulator (in principle, less accurate than an ad hoc MoM) are also included.

Previous sections have reported a procedure to obtain closedform expressions to compute all the parameters involved in the corresponding equivalent networks. Basically, the usage of the equivalent network approach comes down in employing 
the expression of the characteristic TE/TM admittances, transforming the admittances to the discontinuity plane using the well-known procedures of the transmission-line theory, and computing the $N_{h}$ coefficients. In spite of the analytical nature of our proposal, two double infinite series with relatively poor convergence have to be summed up. These series are required to calculate the corresponding capacitance and inductance associated with the contribution of the high-order harmonics. Acceleration techniques developed in the frame of the spectral domain analysis of planar structures can be used to speed up such calculations. However, as previously discussed, these series have to be computed only once for each geometry and thus, even without any numerical treatment, the CPU time required in a standard laptop to compute about the 1000 frequency data points shown in each curve corresponding to our results in this section is below $1 \mathrm{~s}$.

A second important point concerning the final implementation of our method is the current/field spatial profiles employed in the calculations. As our present purpose is to check the validity of the proposed closed-form and self-contained systematic circuit model, we will consider the simple yet practically interesting case of rectangular patches/apertures. Thus, our results in this section have been obtained using the spatial profiles taken from [61] and reported in Appendix A for completitude, which results in a fully analytical model. As discussed in that paper, these simple profiles have proved to be sufficiently accurate to deal with rectangular patches/apertures in many practical situations. Nevertheless, it should be noticed that this is not an intrinsic limitation of the proposed model, since more sophisticated profiles could be used in more complex cases. For scatterers with complex geometry, it can be challenging (if not virtually impossible) to find an approximate analytical expression of the patch current or aperture field. In such cases, however, these profiles could be provided by an external numerical tool (an ad hoc implementation of the MoM or a commercial simulator could be used to this aim). In this way, the external code/simulator would perform the full-wave numerical calculation at a single and relatively low frequency value, and the profile thus obtained used later in the circuit model to find the transmission/reflection spectrum of the structure in a given frequency band and/or for different incidence angles.

The first example shown in Fig. 9 presents our results for a structure previously studied by some of the authors in [47, Fig. 10]. It shows the reflection coefficient for the oblique TE incidence in one of the principal planes of an array of rectangular dipoles printed on a substrate slab. In [47], the authors reported a simplified circuit model whose topology was heuristically obtained and the values of the circuit parameters extracted from a number of previous full-wave simulations carried out at different frequencies. This study is now repeated in Fig. 9 with our results computed following the closed-form expressions given in the present work and using an $x$-directed patch current whose spatial profile is given in (83). Note that the reflection-coefficient curves obtained using the analytical circuit model follow very closely the MoM results in the explored frequency range, which includes a good portion inside the grating lobe regime. In order to give a more quantitative idea on the

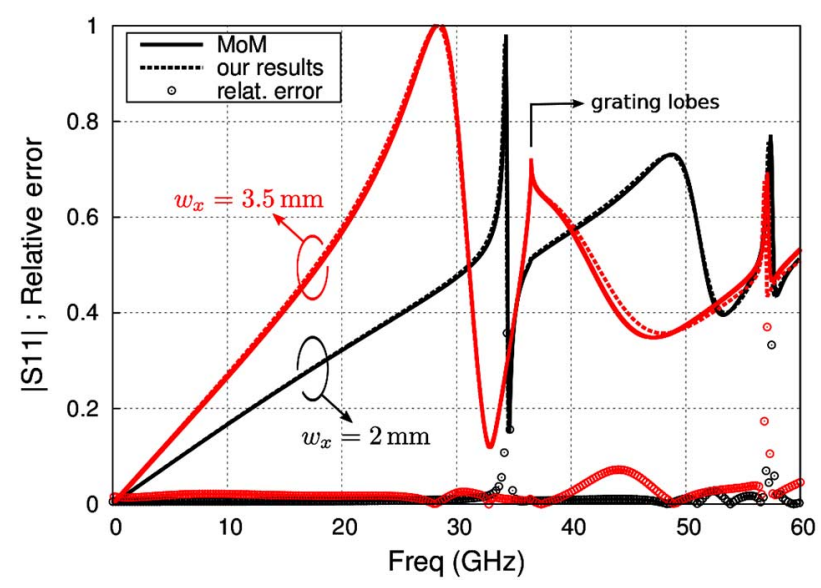

Fig. 9. Magnitude of the reflection coefficient under $40^{\circ}$ TE-incidence in the $y z$ plane $\left(\varphi=90^{\circ}\right)$ for the structure in [47, Fig. 10]. With reference to the top structure in Fig. 8(b): $\varepsilon_{r}^{(2)}=3, d_{1}=0, d_{2}=0.5 \mathrm{~mm}, d_{3}=0, P_{x}=P_{y}=$ $5 \mathrm{~mm}$, and $w_{y}=0.5 \mathrm{~mm}$.

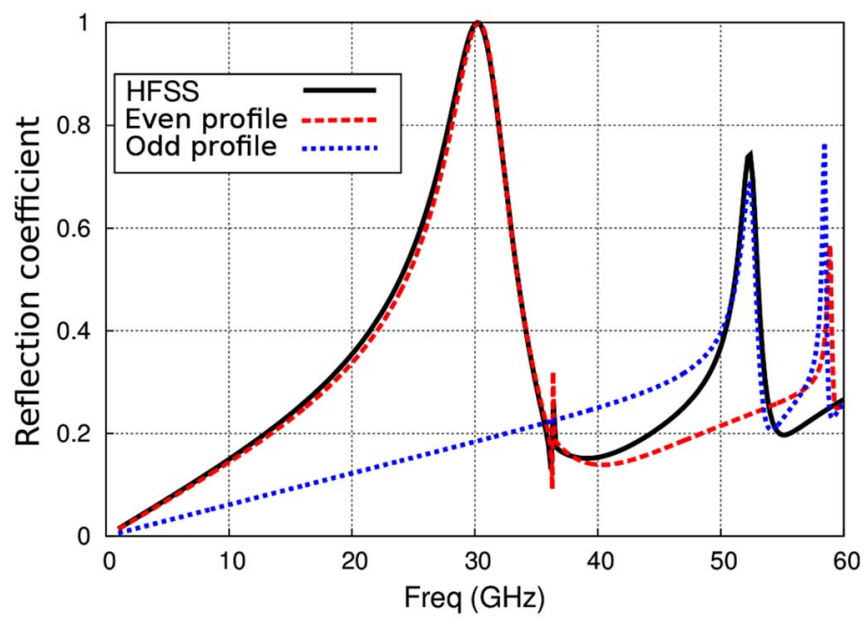

Fig. 10. Same as Fig. 9 with $w_{x}=3.5 \mathrm{~mm}$, but under TM-incidence in the $x z$ plane $\left(\varphi=0^{\circ}\right)$

accuracy of the model, the relative error between both sets of data is also shown. It can be observed that the error remains in the order of a few percent, except for frequencies at which the spectra exhibit narrow peaks. This is somehow expected due to the very fast variation of the magnitudes being compared; nevertheless the frequency shift between the maxima (which constitutes a better indicator of the accuracy under these circumstances) is below $1.5 \%$ for any of the peaks in the plot. (In the following plots, the errors will not be shown explicitly; however, it has been checked that its behavior is very similar to the present case.)

In Fig. 9, it is worth to note that the circuit-model approach perfectly captures the first reflection peak at about $27 \mathrm{GHz}$ for the $w_{x}=3.5$ - $\mathrm{mm}$ case. This peak is clearly associated with the first resonance of the metallic scatterer and is well reproduced by our procedure since the spatial profile of the current in (83) conveniently accounts for the natural half-wavelength-like profile at the first patch resonance. However, higher resonances associated with the scatterer dimensions are not expected to 
be well characterized by the present approach since the current profile associated with these higher resonances strongly differs from that of the first resonance. Thus, the analytical model obtained using the proposed "low-frequency" profile is expected to provide accurate results up to frequencies close to the second resonance of the patch. By this reasoning, the $w_{x}=3.5$-mm curve in Fig. 9 should start to deviate considerably from the full-wave MoM data well below $60 \mathrm{GHz}$ (the second resonance of the patch is expected at roughly twice the first resonance frequency). However, this discrepancy is not observed in Fig. 9, given that the second resonance of the patches is not excited in this case due to symmetries of the problem (the middle plane of the dipoles is an electric-wall symmetry plane, which is incompatible with the odd parity of the one-wavelength current profile associated with this resonance). Therefore, the results provided by the circuit model are reliable in all the frequency range shown in the figure and beyond, up to frequencies close to the first higher resonance that is compatible with the incident plane wave.

In order to better illustrate the above discussion on the limitations of the model as well as to further explore its possibilities, Fig. 10 shows the reflection coefficient for the same structure as in Fig. 9 with $w_{x}=3.5 \mathrm{~mm}$, but now the incidence plane is along (instead of perpendicular to) the dipoles. The polarization of the incident wave is changed to TM so that the tangential component of its electric field is still directed along the dipoles. The results provided by our circuit model are shown together with those obtained using the commercial software Ansys HFSS [62]. The curve labeled as "even profile" corresponds to our analytical results obtained using the same patch current profile as in Fig. 9. These analytical results accurately reproduce the first resonance of the patches (reflection peak at about $30 \mathrm{GHz}$ ), but beyond $40 \mathrm{GHz}$ they clearly depart from the HFSS curve and fail to capture the second reflection peak at about $52 \mathrm{GHz}$. This second reflection peak is associated with the second resonance of the patch, whose excitation is not inhibited now by the incident wave. The curve labeled as "odd profile" represents the analytical results obtained by using an $x$-directed patch current with an odd $x$-profile given by (85) (this profile closely resembles the one-wavelength profile characteristic of the second resonance of the patch). The results obtained using this odd profile are not valid at lower frequencies, as expected, but they clearly reproduce the second resonance transmission peak. The fact that they are reliable only in a relatively narrow frequency range around the second transmission peak is a clear indication that, in this case and above $40 \mathrm{GHz}$, the patch current can no longer be considered to have a frequency-independent spatial profile over this high frequency band.

A second example corresponds to a square lattice of relatively large (w.r.t. the unit cell) square conducting patches printed on a lossy and conductor backed substrate. Plots for the reflection coefficient of this structure (TE/TM polarization under oblique incidence in the principal planes) have recently been reported in [15, Fig. 15]. Because of the presence of dielectric losses in the grounded substrate, the magnitude of the reflection coefficient shows a minimum at some specific frequency (this minimum would not appear in the absence of

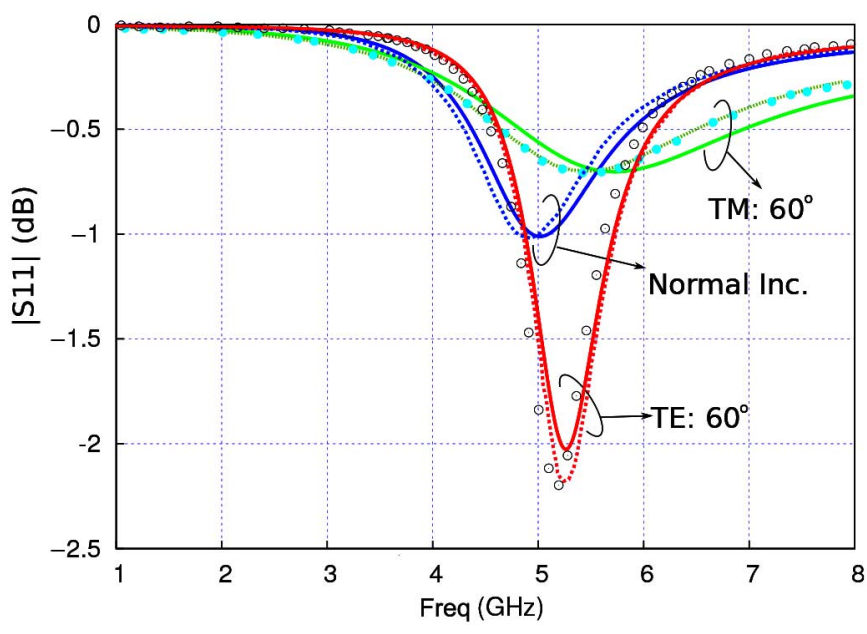

Fig. 11. Magnitude of the reflection coefficient under $60^{\circ}$ TE/TM-incidence $\left(\varphi=90^{\circ}\right)$ for the reflectarray previously considered in [15, Fig. 15]. With reference to the top structure in Fig. $8(\mathrm{~b}), \varepsilon_{r}^{(2)}=4.5-$ j0.088, $d_{1}=0$, $d_{2}=3 \mathrm{~mm}, d_{3}=0, P_{x}=P_{y}=10 \mathrm{~mm}$, and $w_{x}=w_{y}=14 / 16 P_{x}$. In this structure, there is a ground plane backing medium (2). Solid lines: our circuit-model results. Dotted lines: MoM results. Symbols: data of the circuit model in [15].

losses, of course). In Fig. 11, our analytical results are compared with the data in [15, Fig. 15]. This figure shows an acceptable agreement between our results and those reported in that paper.

The case of a slot-based FSS is illustrated in Fig. 12. The structure considered in that figure is a periodically perforated metallic screen (rectangular slots) backed by an electrically thick silicon substrate (see Fig. 12(a)]. Fig. 12(b) shows the frequency response of the magnitude of the transmission coefficient of a TM plane wave that impinges obliquely on the FSS $\left(\theta=20^{\circ}, \varphi=90^{\circ}\right)$. This situation was previously studied in [12, Fig. 4], where the authors reported experimental results showing an excellent agreement with numerical calculations based on the highly accurate and efficient MoM/BI-RME. Fig. 12(b) only includes the numerical data in [12, Fig. 4] and our analytical results ( $M=1$ has been chosen for both TE and TM contributions, i.e., only the distributed nature of the first TE and TM high-order modes has been retained in the computations, with the contribution of the remaining high-order modes being subsumed in the lumped capacitor and inductor). The agreement between the reported results in [12] and our circuit-model results is very good, even in the fine details of this complicated spectrum. Also, this structure has been chosen to illustrate how the circuit model can help us to understand the frequency response and to design the device. To that end, a more detailed study of the application of the circuit models to this structure is shown in Appendix B, which is based on the minimal circuit models that yield a physically sensible and reasonably accurate response. A similar discussion can be done for any other given structure, but this is beyond the aim of the present "general-purpose" work.

A case of a patch-based FSS embedded in a three-layer dielectric environment as in Fig. 8(b) is next considered in Fig. 13. Our results are here compared with Ansys HFSS [62]. The agreement between the two set of results is very good in 

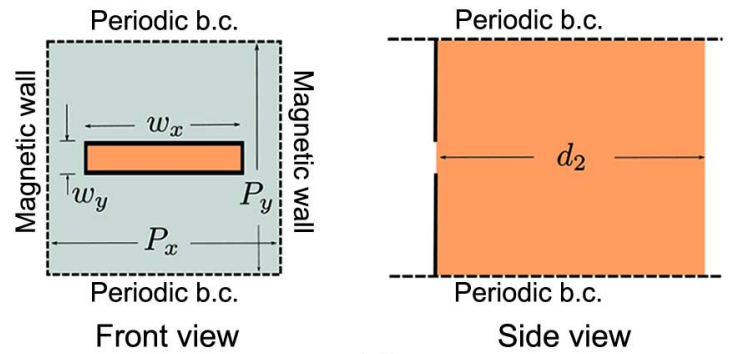

(a)

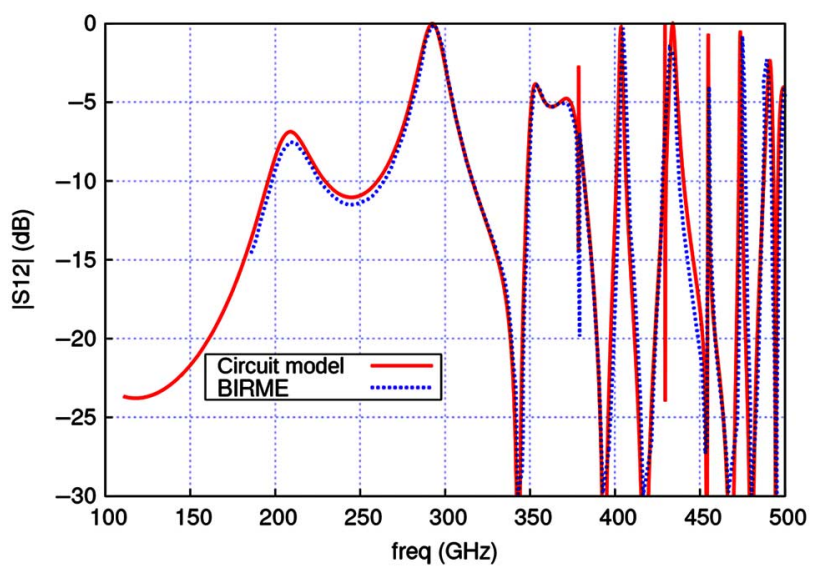

(b)

Fig. 12. (a) Front and side views of the periodically perforated metallic screen printed on a silicon substrate previously considered in [12, Fig. 4]. (b) Magnitude of the transmission coefficient under $20^{\circ}$ TM-incidence $(\varphi=$ $\left.90^{\circ}\right)$. Structural parameters: $\varepsilon_{r}^{(2)}=11.8, d_{1}=0, d_{2}=302 \mu \mathrm{m}, d_{3}=0$, $P_{x}=P_{y}=236 \mu \mathrm{m}$ and $w_{x}=183 \mu \mathrm{m}, w_{y}=30 \mu \mathrm{m}$. Full-wave results in [12] are here denoted as "BIRME." Circuit model results have been obtained using $M=1$ for both TE and TM harmonics. More details on the application of the circuit model to this structure are given in Appendix B.

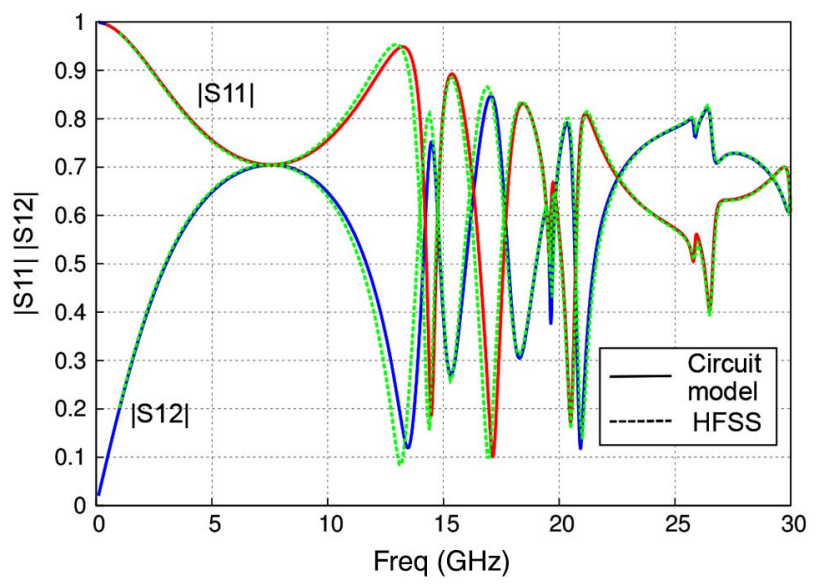

Fig. 13. Magnitude of the reflection and transmission coefficients for the normal incidence of a TM plane wave for an array of rectangular patches with $P_{x}=P_{y}=10 \mathrm{~mm}, w_{x}=4 \mathrm{~mm}, w_{y}=2 \mathrm{~mm}$ embedded in a layered dielectric environment as in Fig. 8(b) with $d_{1}=1 \mathrm{~mm}, d_{2}=2.4 \mathrm{~mm}$, $d_{3}=1 \mathrm{~mm}$, and $\varepsilon_{r}^{(1)}=2.0, \varepsilon_{r}^{(2)}=4.4-\mathrm{j} 0.088, \varepsilon_{r}^{(3)}=10.2$.

the frequency range considered (the grating-lobe regime starts at $30 \mathrm{GHz}$ ). This shows that our approach to deal with a layered dielectric medium works properly. Certainly, the presence of several dielectric layers brings a considerable additional

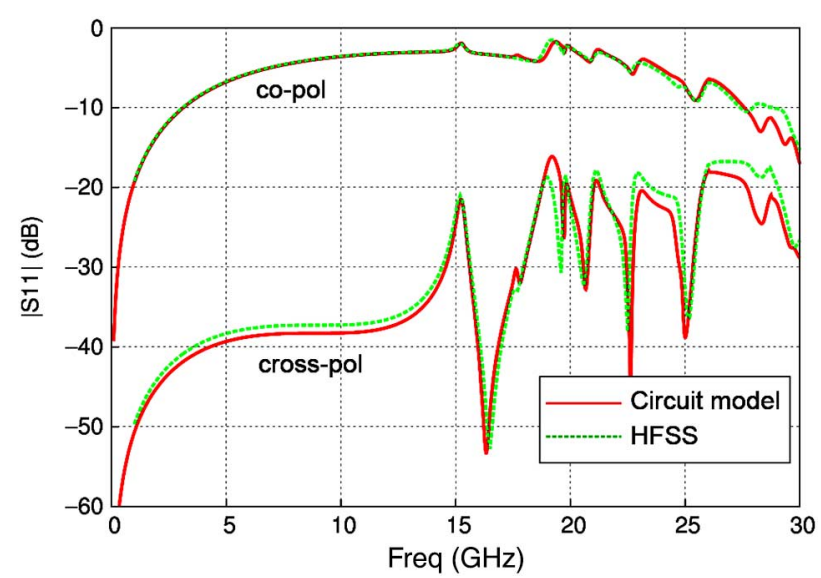

Fig. 14. Magnitude of the co-pol and cross-pol transmission coefficients under conical TE-incidence with $\theta=30^{\circ}, \varphi=60^{\circ}$ for an array of rectangular patches with $\varepsilon_{r}^{(2)}=4.4-\mathrm{j} 0.088, d_{1}=0, d_{2}=2.4 \mathrm{~mm}, d_{3}=0, P_{x}=$ $P_{y}=10 \mathrm{~mm}, w_{x}=4 \mathrm{~mm}$, and $w_{y}=2 \mathrm{~mm}$.

numerical burden for most of commercial electromagnetic simulators. In our approach, these additional dielectric layers hardly affect the computational cost of the presented closedform procedure.

As a final example, a case of conical incidence is considered in Fig. 14 (the incidence plane is no longer directed along the main axes of the structure). In this figure, the magnitude of the reflection coefficient for a FSS under TE illumination is plotted as a function of the frequency. Both the co-pol and cross-pol coefficients are shown. The numerical results needed for comparison have been generated using HFSS [62]. This figure clearly shows that the decomposition procedure followed in this paper to deal with conical incidence works satisfactorily over a wide frequency region (it should be noted that the onset of the diffraction regime is at about $15 \mathrm{GHz}$ ). Moreover, crosspol data are very well predicted by the circuit model in spite of being at a very low power level. Small but significant discrepancies between simulated and analytical data can be appreciated above $26 \mathrm{GHz}$. But the electrical size of the rectangular patch in this range of frequencies is well above half-wavelength, in such a way that the current distribution profile starts to be frequencydependent. Even in this latter frequency region, the analytical model still gives a reasonable approximation to the response calculated by means of a highly intensive numerical code.

\section{CONCLUSiON}

An equivalent multimode transmission-line network is formally derived for the scattering problem posed by the incidence of a plane wave on a 2-D array of metal patches printed on (or embedded in) a stratified dielectric environment. The quasidual structure consisting of a 2-D array of rectangular slots etched in a metal film coated with dielectric slabs has been treated in a similar manner. Both TE and TM polarizations (with respect to the incidence plane) and arbitrary angles of incidence (conical incidence) are considered in the theoretical formulation. The attained solutions lead themselves to equivalent circuit models involving lumped capacitors, inductors, and transmission line sections. All the parameters of the equivalent 
circuit are analytically known, provided a reasonable guess for the field distribution in the apertures or the current distribution on the metal patches is available. The reflection and transmission coefficients obtained with the circuit model are not very sensitive to the details of the aforementioned field/current distributions. A key feature of the proposed circuit model when compared with other alternatives is that the circuit topology has been derived directly from the fundamental integral equations associated with the electromagnetic problem. Moreover, dynamic effects that are not incorporated in other circuit models are included in the proposed model thanks to the differentiated treatment of low- and high-order scattered space harmonics. In this way, the frequency-dependent contribution of the first few modes is explicitly extracted out, while the remaining higher order modes contribution is represented by frequencyindependent capacitors or inductors. The accuracy and suitability of the model has been satisfactorily proved through a number of comparisons with numerically generated data.

\section{APPENDIX A}

For the cases studied in Fig. 9, Fig. 10 (even profile), TE incidence in Fig. 11, and Fig. 13, we used an $x$-directed patch current whose spatial profile is given by

$$
f_{\mathrm{e} 1}(x, y)=\cos \left(\frac{\pi x}{w_{x}}\right)\left[1-\left(\frac{2 x}{w_{x}}\right)^{2}\right]^{-1 / 2} \operatorname{rect}\left(\frac{y}{w_{y}}\right) \text {. }
$$

This same spatial profile is used for the $y$-directed aperture electric field in Fig. 12(b). For the TM incidence case in Fig. 11, we used a $y$-directed patch current whose spatial profile is given by (83) but interchanging the roles of $x, w_{x}$ and $y, w_{y}$, namely

$$
f_{\mathrm{e} 2}(x, y)=\cos \left(\frac{\pi y}{w_{y}}\right)\left[1-\left(\frac{2 y}{w_{y}}\right)^{2}\right]^{-1 / 2} \operatorname{rect}\left(\frac{x}{w_{x}}\right) \text {. }
$$

The odd profile used for $J_{x}$ in Fig. 10 is

$$
f_{\mathrm{o}}(x, y)=\sin \left(\frac{2 \pi x}{w_{x}}\right)\left[1-\left(\frac{2 x}{w_{x}}\right)^{2}\right]^{-1 / 2} \operatorname{rect}\left(\frac{y}{w_{y}}\right) \text {. }
$$

Finally, in the conical incidence case in Fig. 14, we need two different patch current profiles, according to Section IV. Thus, for the $x$-problem, we use an $x$-directed patch current with the spatial profile given by (83); whereas for the $y$-problem, we used a $y$-directed current with the profile in (84).

\section{APPENDIX B}

This Appendix will show how the equivalent-circuit approach described in this work explains, in a simple manner, the observed transmission and reflection spectra and how it can help in the design process. Let us take into consideration the periodic structure studied in Fig. 12, which is one of the structures analyzed in [12]. This filtering device consists of a 2-D square periodic distribution of narrow slits made in a thin metallic layer. This layer is printed on an electrically thick silicon substrate that strongly modifies the response of the perforated metal surface. The structure is obliquely illuminated $\left(\theta=20^{\circ}, \varphi=90^{\circ}\right)$ by a TM-polarized plane wave. The simplest and most intuitive equivalent circuit for this structure is shown in Fig. 15(a). This is the kind of equivalent circuit typically used in the literature to handle this type of structures. The $L C$-tank should account for the effect of the scattered high-order harmonics and the transmission line sections would account for the TEM propagating fundamental mode. The values of the parameters $L_{\mathrm{ho}}$ and $C_{\mathrm{ho}}$ are computed using (47) and (48) with $(n, m) \neq(0,0)$. The line characteristic admittances and propagation constants are given by (13) taking $n, m=0$. The magnitude of the transmission coefficient $\left(\left|S_{12}\right|\right)$ obtained with this simplified analytical model is plotted in Fig. 16 as a red-color curve. By comparing this curve with the results in Fig. 12(b), it is clear that the prediction of this simplified circuit model is quite accurate in the low-frequency regime (roughly speaking, below $220 \mathrm{GHz}$ ), but it progressively deteriorates for higher frequencies. Nevertheless, the second transmission peak at $294 \mathrm{GHz}$ in Fig. 12(b) still appears in the red-color transmission curve in Fig. 16 although strongly blue-shifted (it now appears at $318 \mathrm{GHz}$, which means more than $8 \%$ error). The fact that this second peak shows an apparent total transmissivity can be easily understood from the circuit in Fig. 15(a). A trivial circuit rationale tells us that in order to avoid reflection at a given frequency, the normalized input admittance $Y_{\text {in }} / Y_{0}^{(0)}$ has to be as close to unity as possible. This condition can be satisfied if the electrical length of the transmission line corresponding to the silicon region is close to $n \pi / 2$ ( $n$ being a positive integer number) and, simultaneously, the slit is resonating (the $L C$ circuit would then behave as an open circuit). The slit resonance should occur at the frequency for which the length of the slit is $\lambda_{\text {eff }} / 2$, with $\lambda_{\text {eff }}$ being the wavelength in the effective medium whose permittivity corresponds to the average value of the dielectric constants of air and silicon (this is a good approximation since the silicon layer is relatively thick); namely, $\varepsilon_{\text {eff }}=(11.8+1) / 2=6.4$ in our case. This resonance condition is found at $314.2 \mathrm{GHz}$. At such frequency, the thickness of the silicon layer is about 1.082 times the wavelength in the transmission line representing such layer (the effect of the incidence angle $\theta=20^{\circ}$, although small, is incorporated in this calculation). The real and imaginary parts of the normalized load admittance predicted by the circuit in Fig. 15(a) are plotted in Fig. 17 (red curves). Note that the best matching happens at about $317.5 \mathrm{GHz}$, frequency at which the imaginary part of the input admittance vanishes.

Although the above given explanation is qualitatively satisfactory, the shift of the resonance frequency is too large to be considered acceptable from a practical point of view. However, this lack of accuracy can be anticipated in the frame of our modeling technique. Indeed, the problem comes from the fact that the cutoff frequency of the first high-order harmonic involved in the scattered field is not far enough from the second transmission peak. A simple calculation for the first relevant harmonic 


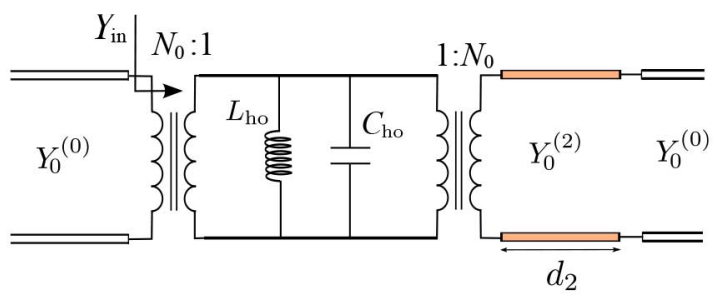

(a)
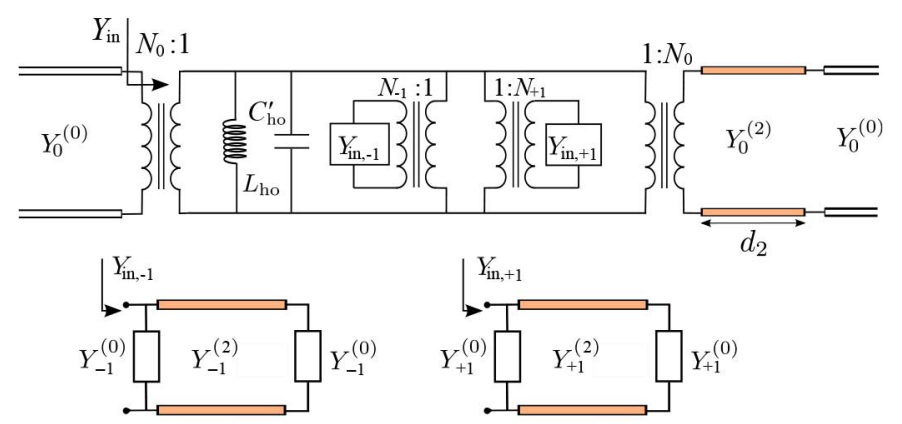

(b)

Fig. 15. (a) Standard circuit model for the study of the scattering of the fundamental harmonic. The contribution of the high-order diffracted harmonics are accounted for by the frequency-independent lumped components $C_{\mathrm{ho}}$ and $L_{\mathrm{ho}}$. (b) Enhanced circuit model that takes into account the distributed effects of the first high-order TM harmonics $(n=0, m= \pm 1)$. The characteristic admittances and propagation constants of the transmission line sections are the ones corresponding to the involved harmonics as defined in (13)-(16). Although the effect of the first TE harmonics is much weaker, their distributed nature was also considered in the results shown in Fig. 12(b).

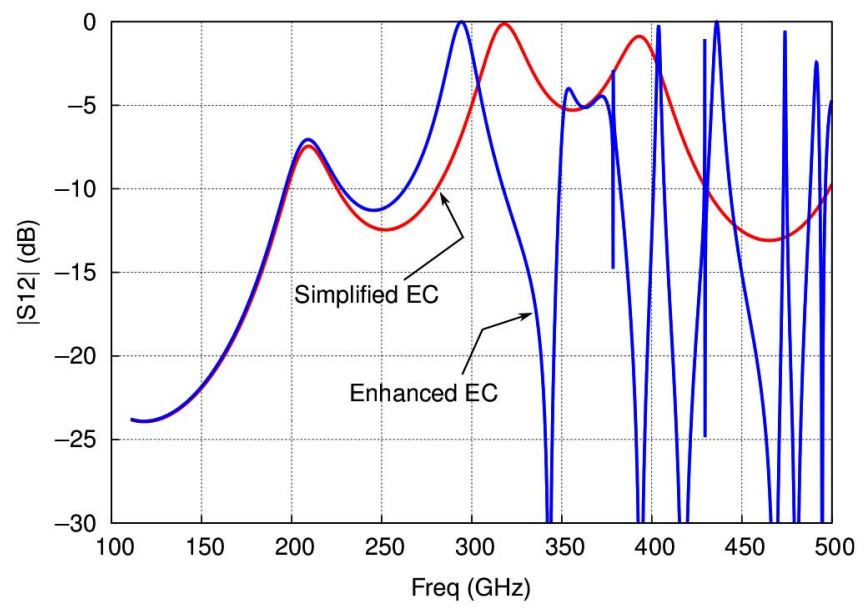

Fig. 16. Transmission coefficient of the structure analyzed in Fig. 12 using the model in Fig. 15(a) (red line) and the enhanced version (blue line) of this model in Fig. 15(b).

$\left(\mathrm{TM}_{0,-1}\right)$ yields a cutoff frequency of $336.56 \mathrm{GHz}$. It means that the circuit model should take into account the distributed nature of this harmonic. Thus, its contribution should not be included in the capacitance $C_{\mathrm{ho}}$ of the model in Fig. 15(a), but implemented as a distributed transmission line, as shown in Fig. 15(b). Since the cutoff frequency of the $\mathrm{TM}_{0,+1}$ harmonic is $410.99 \mathrm{GHz}$ and we are interested on the frequency response up to $500 \mathrm{GHz}$, this $m=+1 \mathrm{TM}$ harmonic is also considered in the same way. The analytically obtained response

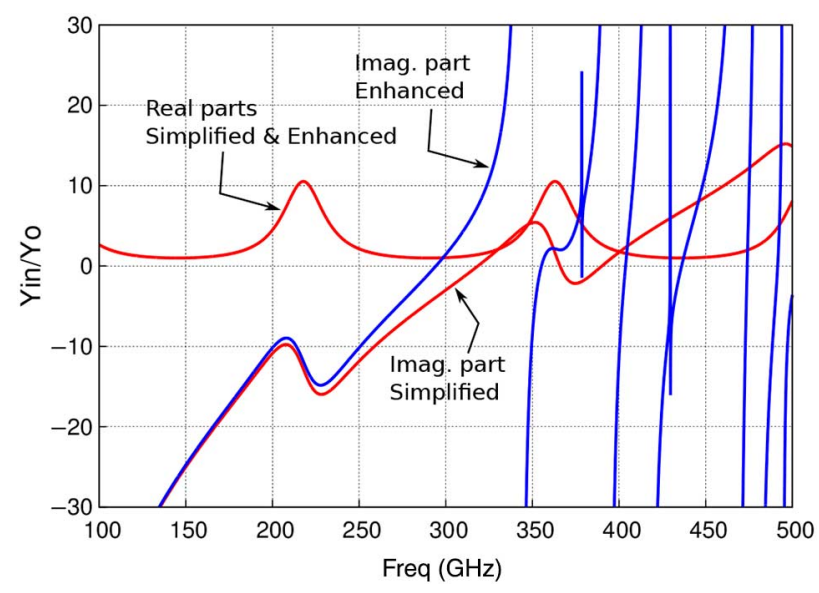

Fig. 17. Real and imaginary parts of the input admittance loading the input transmission line of our circuit models. The red line corresponds to the simplified standard circuit in Fig. 15(a) and the blue line is the one corresponding to the enhanced circuit model in Fig. 15(b). The real part is the same for both cases.

that results after these considerations is shown in Fig. 16 in blue. The highly accurate numerical results in Fig. 12(b) are now analytically reproduced with a much smaller quantitative error (the total transmission resonance peak is only $2.2 \%$ blue-shifted now). Moreover, the complicated high-frequency response above the second peak is much better reproduced. At the new resonance frequency of $294.3 \mathrm{GHz}$, the thickness of the silicon layer is only 1.012 times the silicon transmission line wavelength, which is much closer to the perfect transmission condition than the prediction of the simplified model. Actually, at $294.3 \mathrm{GHz}$, the imaginary part of the complex admittance loading the input transmission line in the model in Fig. 15(b) vanishes and the computed real part is 1.0063 . A plot of the imaginary part of the normalized load impedance versus frequency is shown as a blue curve in Fig. 17. Note that multiple zeros and peaks appear above $340 \mathrm{GHz}$ that were absent in the simplified conventional circuit model. These can actually be associated with the behavior of the first high-order TM harmonic, which is a propagating wave in the dielectric region above $336.56 \mathrm{GHz}$. There is a clear correspondence between the transmission zeros in Fig. 16 and the singularities of the normalized admittance in Fig. 17. This way, the analytical circuit model in Fig. 15(b) provides much more valuable information on the electrical response of the structure under study than the straightforward usual approach represented by the circuit in Fig. 15(a). Although less relevant than the above TM harmonics, the $m= \pm 1$ TE harmonics also play some role in the structure response within the considered frequency range. Thus, they can also be explicitly treated as distributed contributions in the same manner. By doing this, the curves reported in Fig. 12(b) are obtained, which are indistinguishable from the full-wave numerical data.

\section{ACKNOWLEDGMENT}

The authors would like to thank M. García-Vigueras for her valuable contribution to the initial developments of the present work. 


\section{REFERENCES}

[1] G. G. Macfarlane, "Quasi-stationary field theory and its application to diaphragms and junctions in transmission lines and wave guides," J. Inst. Electr. Eng. Part 3A, vol. 93, no. 4, pp. 703-719, Apr. 1946.

[2] J. Brown, "Artificial dielectrics having refractive indices less than unity," Proc. IEE Part IV Inst. Monogr., vol. 100, no. 62R, pp. 51-62, Oct. 1953.

[3] G. V. Trentini, "Partially reflecting sheet arrays," IRE Trans. Antennas Propag., vol. 4, pp. 666-671, 1956.

[4] C.-C. Chen, "Diffraction of electromagnetic waves by a conducting screen perforated periodically with circular holes," IEEE Trans. Microw. Theory Techn., vol. 19, no. 5, pp. 475-481, May 1971.

[5] C.-C. Chen, "Transmission of microwave through perforated flat plates of finite thickness," IEEE Trans. Microw. Theory Techn., vol. 21, no. 1, pp. 1-6, Jan. 1973.

[6] R. Ulrich, "Far-infrared properties of metallic mesh and its complementary structure," Infrared Phys., vol. 7, pp. 37-55, 1967.

[7] R. Ulrich, "Effective low-pass filters for far infrared frequencies," Infrared Phys., vol. 7, pp. 65-74, 1967.

[8] V. Torres et al., "Mid-infrared plasmonic inductors: Enhancing inductance with meandering lines," Sci. Rep., vol. 4, Art. 3592, Jan. 2014.

[9] J. C. Vardaxoglou, Frequency Selective Surfaces Analysis and Design, 1st Ed. Taunton, MA, USA: Research Studies, 1997.

[10] Z. L. Wang, K. Hashimoto, N. Shinohara, and H. Matsumoto, "Frequency-selective surface for microwave power transmission," IEEE Trans. Microw. Theory Tech., vol. 47, no. 10, pp. 2039-2042, Oct. 1999.

[11] B. Munk, Frequency Selective Surfaces: Theory and Design Hoboken, NJ, USA: Wiley, 2000

[12] S. Biber, M. Bozzi, O. Günther, L. Perregrini, and L.-P. Schmidt, "Design and testing of frequency-selective surfaces on silicon substrates for submillimeter-wave applications," IEEE Trans. Antennas Propag., vol. 54, no. 9, pp. 2638-2645, Sep. 2006.

[13] M. Al-Joumayly and N. Behdad, "A new technique for design of low-profile, second-order, bandpass frequency selective surfaces," IEEE Trans. Antennas Propag., vol. 57, no. 2, pp. 452-459, Feb. 2009.

[14] J. Huang and J. A. Encinar, Reflectarray Antennas. Hoboken, NJ, USA: Wiley/IEEE Press, 2007.

[15] F. Costa and A. Monorchio, "Closed-form analysis of reflection losses in microstrip reflectarray antennas," IEEE Trans. Antennas Propag., vol. 60 , no. 10 , pp. 4650-4660, Oct. 2012.

[16] A. B. Yakovlev, Y. R. Padooru, G. W. Hanson, A. Mafi, and S. Karbasi, "A generalized additional boundary condition for mushroom-type and bedof-nails-type wire media," IEEE Trans. Microw. Theory Tech., vol. 59, no. 3, pp. 527-531, Mar. 2011

[17] A. Foroozesh and L. Shafai, "On the characteristics of the highly directive resonant cavity antenna having metal strip grating superstrate," IEEE Trans. Antennas Propag., vol. 60, no. 1, pp. 78-91, Jan. 2012.

[18] T. W. Ebbesen, H. J. Lezec, H. F. Ghaemi, T. Thio, and P. A. Wolff, "Extraordinary optical transmission through sub-wavelength hole arrays," Nature, vol. 391, pp. 667-669, Feb. 1998.

[19] F. J. García-de-Abajo, "Colloquium: Light scattering by particle and hole arrays," Rev. Mod. Phys., vol. 79, pp. 1267-1290, Oct./Dec. 2007

[20] F. J. García-Vidal, L. Martín-Moreno, T. W. Ebbesen, and L. Kuipers, "Light passing through subwavelength apertures," Rev. Mod. Phys., vol. 82, pp. 729-787, Jan./Mar. 2010.

[21] A. V. Kildishev, A. Boltasseva, and V. M. Shalaev, "Planar photonics with metasurfaces," Science, vol. 339, p. 1232009, Mar. 2013.

[22] T. L. Zinenko, M. Marciniak, and A. I. Nosich, "Accurate analysis of light scattering and absorption by an infinite flat grating of thin silver nanostrips in free space using the method of analytical regularization," IEEE J. Sel. Topics Quantum Electron., vol. 19, no. 3, p. 9000108, May/Jun. 2013.

[23] D. Zhao, H. Gong, Y. Yang, Q. Li, and M. Qiu, "Realization of an extraordinary transmission window for a seamless $\mathrm{Ag}$ film based on metal-insulator-metal structures," Appl. Phys. Lett. vol. 102, p. 201109, May 2013

[24] F. Capolino, Ed. Metamaterial Handbook: Theory and Phenomena of Metamaterials. Boca Raton, FL, USA: CRC Press, 2009.

[25] I. Palocz and A. A. Oliner, "Equivalent network of a multimode planar grating," IEEE Trans. Microw. Theory Tech., vol. 18, no. 5, pp. 244-252, May 1970.

[26] S.-W. Lee, G. Zarrillo, and C.-L. Law, "Simple formulas for transmission through periodic metal grids or plates," IEEE Trans. Antennas Propag., vol. 30, no. 5, pp. 904-909, May 1982.

[27] C. K. Lee and R. J. Langley, "Equivalent-circuit models for frequencyselective surfaces at oblique angles of incidence," IEE Proc. Microw. Antennas Propag. H, vol. 132, no. 6, pp. 395-399, Oct. 1985.
[28] G. Zarrillo and K. Aguiar. "Closed-form low frequency solutions for electromagnetic waves through a frequency selective surface," IEEE Trans. Antennas Propag., vol. 35, no. 12, pp. 1406-1417, Dec. 1987.

[29] M. Guglielmi and A. A. Oliner, "Multimode network description of a planar periodic metal-strip grating at a dielectric interface-Part I: Rigorous network formulations," IEEE Trans. Microw. Theory Tech., vol. 37, no. 3 , pp. 535-541, Mar. 1989.

[30] M. Guglielmi and A. A. Oliner, "Multimode network description of a planar periodic metal-strip grating at a dielectric interface-Part II: Smallaperture and small-obstacle solutions," IEEE Trans. Microw. Theory Tech., vol. 37, no. 3, pp. 542-552, Mar. 1989.

[31] P. Callaghan, E. A. Parker, and R. J. Langley, "Influence of supporting dielectric layers on the transmission properties of frequency selective surfaces," IEE Proc. Microw. Antennas Propag. H, vol. 138, no. 5, pp. 448-454, May 1991.

[32] R. Dubrovka, J. Vazquez, C. Parini, and D. Moore, "Equivalent circuit method for Analysis and synthesis of frequency selective surfaces," IEE Proc. Microw. Antennas Propag. H, vol. 153, no. 3, pp. 213-220, Mar. 2006.

[33] S. Maci, M. Caiazzo, A. Cucini, and M. Casaletti, "A pole-zero matching method for EBG surfaces composed of a dipole FSS printed on a grounded dielectric slab," IEEE Trans. Antennas Propag., vol. 53, no. 1, pp. 70-81, Jan. 2005

[34] S. Monni, G. Gerini, A. Neto, and A. G. Tijhuis, "Multi-mode equivalent networks for the design and analysis of frequency selective surfaces," IEEE Trans. Antennas Propag., vol. 55, no. 10, pp. 2824-2835, Oct. 2007.

[35] O. Luukkonen et al., "Simple and accurate analytical model of planar grids and high-impedance surfaces comprising metal strips or patches," IEEE Trans. Antennas Propag., vol. 56, no. 6, pp. 1624-1632, Jun. 2008.

[36] O. Luukkonen, F. Costa, C. R. Simovski, A. Monorchio, and S. A. Tretyakov, "A thin electromagnetic absorber for wide incidence angles and both polarizations," IEEE Trans. Antennas Propag., vol. 57, no. 10, pp. 3119-3125, Oct. 2009.

[37] Y. R. Padooru et al., "New absorbing boundary conditions and analytical model for multilayered mushroom-type metamaterials: Applications to wideband absorbers," IEEE Trans. Antennas Propag., vol. 60, no. 12, pp. 5727-5742, Dec. 2012

[38] J. W. Miles, "The equivalent circuit for a plane discontinuity in a cylindrical wave guide," Proc. IRE, vol. 34, no. 10, pp. 728-742, Oct. 1946.

[39] N. Marcuvitz, Waveguide Handbook, vol. 10, New York, NY, USA: McGraw-Hill, 1951. New Ed., Stevenage, U.K.: IEE Publishing/Peregrinus, 1986.

[40] L. B. Felsen and A. A. Oliner, "Determination of equivalent circuit parameters for dissipative microwave structures," Proc. IRE, vol. 42, no. 2, pp. 477-483, Feb. 1954.

[41] A. Wexler, "Solution of waveguide discontinuitites by modal analysis," IEEE Trans. Microw. Theory Tech., vol. MTT-15, no. 9, pp. 508-517, Sep. 1967.

[42] J. Schwinger and D. S. Saxon, Discontinuities in Waveguides. New York, NY, USA: Gordon and Breach, 1968.

[43] F. Medina, F. Mesa, and R. Marqués, "Extraordinary transmission through arrays of electrically small holes from a circuit theory perspective," IEEE Trans. Microw. Theory Tech., vol. 56, no. 12, pp. 3108-3120, Dec. 2008.

[44] M. Beruete, M. Navarro-Cía, and M. Sorolla, "Understanding anomalous extraordinary transmission from equivalent circuit and grounded slab concepts," IEEE Trans. Microw. Theory Tech., vol. 59, no. 9 , pp. 2180-2188, Sep. 2011.

[45] R. Rodriguez-Berral, F. Medina, F. Mesa, and M. Garcia-Vigueras, "Quasi-analytical modeling of transmission/reflection in strip/slit gratings loaded with dielectric slabs," IEEE Trans. Microw. Theory Tech., vol. 60, no. 3, pp. 405-418, Mar. 2012.

[46] F. Costa, A. Monorchio, and G. Manara, "Efficient analysis of frequencyselective surfaces by a simple equivalent-circuit model," IEEE Antennas Propag. Mag., vol. 54, no. 4, pp. 35-48, Aug. 2012.

[47] M. García-Vigueras, F. Mesa, F. Medina, R. Rodríguez-Berral, and J. L. Gómez-Tornero, "Simplified circuit model for metallic arrays of patches sandwiched between dielectric slabs under arbitrary incidence," IEEE Trans. Antennas Propag., vol. 60, no. 10, pp. 4637-4649, Oct. 2012.

[48] F. Capolino, A. Vallecchi, and M. Albani, "Equivalent transmission line model with a lumped X-circuit for a metalayer made of pairs of planar conductors," IEEE Trans. Antennas Propag., vol. 61, no. 2, pp. 852-861, Feb. 2013 
[49] F. Costa, S. Genovesi, A. Monorchio, and G. Manara, "A circuit-based model for the interpretation of perfect metamaterial absorbers," IEEE Trans. Antennas Propag., vol. 61, no. 3, pp. 1201-1209, Mar. 2013.

[50] F. Costa, S. Genovesi, and A. Monorchio, "A chipless RFID based on multiresonant high-impedance surfaces," IEEE Trans. Microw. Theory Tech., vol. 61, no. 1, pp. 146-153, Jan. 2013.

[51] V. Lomakin and E. Michielssen, "Enhanced transmission through metallic plates perforated by arrays of subwavelength holes and sandwiched between dielectric slabs," Phys. Rev. B, vol. 71, p. 235117, Jun. 2005.

[52] R. Ortuño, C. García-Meca, F. J. Rodríguez-Fortuo, J. Martí, and A. Martínez, "Multiple extraordinary optical transmission peaks from evanescent coupling in perforated metal plates surrounded by dielectrics," Opt. Express, vol. 18, no. 8, pp. 7893-7898, Apr. 2010.

[53] C. S. R. Kaipa, A. B. Yakovlev, S. I. Maslovski, and M. G. Silveirinha, "Mushroom-type high-impedance surface with loaded vias: Homogenization model and ultra-thin design," IEEE Antennas Wireless Propag. Lett., vol. 10, pp. 1503-1506, Feb. 2012.

[54] S. A. Tretyakov, Analytical Modeling in Applied Electromagnetics. Norwood, MA, USA: Artech House, 2003.

[55] R. Rodríguez-Berral, C. Molero, F. Medina, and F. Mesa, "Analytical wideband model for strip/slit gratings loaded with dielectric slabs," IEEE Trans. Microw. Theory Tech., vol. 60, no. 12, pp. 3908-3918, Dec. 2012.

[56] J. L. Tsalamengas, "Scattering of arbitrarily polarized plane waves obliquely incident on infinite slots or strips in a planar-stratified medium," IEEE Trans. Antennas Propag., vol. 46, no. 11, pp. 1634-1640, Nov. 1998.

[57] R. Florencio, R. R. Boix, and J. A. Encinar, "Enhanced MoM analysis of the scattering by periodic strip gratings in multilayered substrates," IEEE Trans. Antennas Propag., vol. 61, no. 10, pp. 5088-5099, Oct. 2013.

[58] R. Rodríguez-Berral, F. Mesa, F. Medina, and M. García-Vigueras, "Analytical circuit model for dipole frequency-selective surfaces," in Proc. Int. Microw. Symp. (IMS'13), Seattle, WA, USA, Jun. 2013, pp. $1-4$.

[59] J. E. Varela and J. Esteban, "Characterization of waveguides with a combination of conductor and periodic boundary contours: Application to the analysis of bi-periodic structures," IEEE Trans. Microw. Theory Tech., vol. 60, no. 3, pp. 419-430, Mar. 2012.

[60] D. M. Pozar, Microwave Enginnering, 3rd Ed. Hoboken, NJ, USA: Wiley, 2005.

[61] S. R. Rengarajan, "Choice of basis functions for accurate characterization of infinite array of microstrip reflectarray elements," IEEE Antennas Wireless Propag. Lett., vol. 4, pp. 47-50, Jun. 2005.

[62] ANSYS. High Frequency Structure Simulator (HFSS) [Online] Available: http://www.ansys.com/Products/Simulation+Technology/ Electromagnetics/High-Performance+Electronic+Design/ANSYS+HFSS

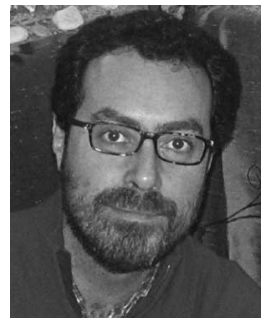

Raúl Rodríguez-Berral was born in Casariche, Seville, Spain, in 1978. He received the M.Sc. (Licenciado) and Ph.D. degrees in physics from the University of Seville, Seville, Spain, in 2001 and 2008, respectively.

In January 2002, he joined the Department of Applied Physics 1, University of Seville, where he is currently an Associate Professor. His research interests include the study of the spectrum and the excitation of periodic and nonperiodic planar structures and high-frequency circuit modeling.

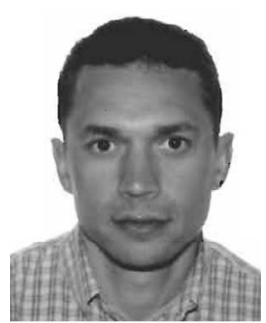

Francisco Mesa (M'93-SM'11-F'14) was born in Cádiz, Spain, in April 1965. He received the Licenciado and Doctor degrees in physics from the Universidad de Sevilla, Seville, Spain, in 1989 and 1991, respectively.

He is currently a Professor with the Departamento de Física Aplicada 1, Universidad de Sevilla, Seville, Spain. His research interests include electromagnetic propagation/radiation in planar structures.

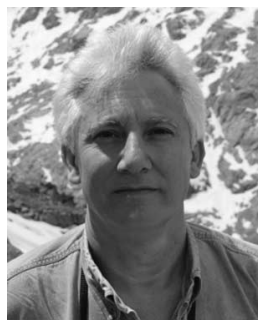

Francisco Medina (M'90-SM'01-F'10) was born in Puerto Real, Cádiz, Spain, in November 1960. $\mathrm{He}$ received the Licenciado and Doctor degrees in physics from the University of Seville, Seville, Spain, in 1983 and 1987 respectively.

$\mathrm{He}$ is currently a Professor of Electromagnetism with the Department of Electronics and Electromagnetism, University of Seville, and Head of the Microwaves Group. He has co-authored more than 130 journal papers and book chapters on those topics and more than 260 conference contributions. His research interests include analytical and numerical methods for planar structures, anisotropic materials, and artificial media modeling.

Dr. Medina acts as Reviewer for more than 40 IEEE, IET, AIP, and IOP journals and has been a member of the TPCs of a number of local and international conferences. 